\title{
COUNTABLE ULTRAHOMOGENEOUS UNDIRECTED GRAPHS ${ }^{1,2}$
}

BY

\author{
A. H. LACHLAN ${ }^{3}$ AND ROBERT E. WOODROW
}

\begin{abstract}
Let $G=\left\langle V_{G}, E_{G}\right\rangle$ be an undirected graph. The complementary graph $\tilde{G}$ is $\left\langle V_{G}, E_{\tilde{G}}\right\rangle$ where $\left(V_{1}, V_{2}\right) \in E_{\tilde{G}}$ iff $V_{1} \neq V_{2}$ and $\left(V_{1}, V_{2}\right) \notin E_{G}$. Let $K(n)$ be the complete undirected graph on $n$ vertices and let $E$ be the graph

i.e. $\langle\{a, b, c\},\{(b, c),(c, b)\}\rangle . G$ is ultrahomogeneous just in case every isomorphism of subgraph of smaller cardinality can be lifted to an automorphism of $G$. Let $\mathscr{T}=\{K(n): n \in \omega\} \cup\{E, \tilde{E}\} \cup\{K(n): n \in \omega\}$. Theorem: Let $G_{1}, G_{2}$ be two countable (infinite) ultrahomogeneous graphs such that for each $H \in \Phi H$ can be embedded in $G_{1}$ just in case it can be embedded in $G_{2}$. Then $G_{1} \simeq G_{2}$. Corollary: There are a countable number of countable ultrahomogeneous (undirected) graphs.
\end{abstract}

0. Introduction and preliminaries. A graph $G$ is a pair $\left\langle|G|, E_{G}\right\rangle$ where $|G|$ is the underlying or vertex set and $E_{G}$ is a binary relation on $|G|$ called the edge set. A graph $G$ is undirected just in case $E_{G}$ is symmetric and irreflexive. Where no confusion is likely to arise we make no distinction between $G$ and $|G|$. If $X$ is a set by $|X|$ we denote the cardinality of $X$. Countable means countable and infinite. Thus the cardinality of a graph $G$ is $\|G\|$, which we write simply $|G|$. We say $H$ is a subgraph of $G$, written $H \subset G$ if $|H| \subset|G|$ and $E_{H}=(|H| \times|H|) \cap E_{G}$. $G$ is ultrahomogeneous just in case each isomorphism $f: H \rightarrow K$ of subgraphs $H, K$ of $G$ with $|H|<|G|$ extends to an automorphism $g: G \rightarrow G(f \subset g)$. This is the notion discussed by Gardiner in [G] where he characterizes the finite undirected ultrahomogeneous graphs.

Ultrahomogeneous relational structures were introduced by Fraissé [F] where they are called simply homogeneous structures. Henson showed in [H1] that there are $2^{\kappa_{0}}$ countable asymmetric homogeneous graphs and introduced the class of countable homogeneous undirected graphs; in [H2] he explores further the properties of these structures. Schmerl [S] has shown that there are a countable number of countable homogeneous partial orderings. Peretyiatkin [P] has also considered homogeneous structures and shows (independently) the existence of $2^{\mathrm{k}_{0}}$ homogeneous directed graphs.

Presented to the Society March 8, 1977; received by the editors August 19, 1977 and, in revised form, February 15, 1979.

1980 Mathematics Subject Classification. Primary 03C15; Secondary 03C10, 05 C99.

Key words and phrases. Homogeneous, ultrahomogeneous, undirected graph, elimination of quantifiers, amalgamation property, $\aleph_{0}$-categorical.

${ }^{1}$ The authors express their thanks to Mr. Ron Morrow for his time spent in discussion of this work and his contributions to the proofs of Lemmas 1 and 3.

${ }^{2}$ This work was supported in part by NRC Grant no. 741003.

${ }^{3}$ The first author is grateful for the hospitality of the Institute of Mathematics of the University of Warsaw where part of this work was done. 
We came upon this problem via the work of Gardiner. He uses "homogeneous graph" in a different sense than Fraissé and others. In [G] a graph is homogeneous provided that whenever $H_{1}, H_{2}$ are isomorphic subgraphs of smaller cardinality there is some automorphism which carries $H_{1}$ onto $H_{2}$. Because "ultrahomogeneous" has been used in only the one sense we adopt its use.

Throughout the rest of the paper we shall be solely concerned with undirected graphs. Let $K(n)$ be the complete graph with $n$ vertices for $n \geqslant 1$. In [W1] Woodrow described those countable ultrahomogeneous graphs in which the triangle $K(3)$ cannot be embedded, and conjectured that there are only finitely many countable ultrahomogeneous graphs in which $K(4)$ cannot be embedded. In [W2] he conjectured that for each $n$ there are a finite number of countable ultrahomogeneous graphs in which $K(n)$ cannot be embedded. In this paper a new approach by the first author allows the methods of [W1] to be extended to characterize all the countable ultrahomogeneous graphs, thus verifying the conjectures just mentioned. We shall use certain of the results of [W1] which we now list.

Let $\Sigma$ be a class of graphs. We say that $\Sigma$ has the amalgamation property (AP) just in case whenever $A, B_{0}, B_{1} \in \Sigma$, and $f_{0}: A \rightarrow B_{0}, f_{1}: A \rightarrow B_{1}$ are embeddings then there is $C \in \Sigma$ and there are embeddings $g_{0}: B_{0} \rightarrow C, g_{1}: B_{1} \rightarrow C$ such that $g_{0} \circ f_{0}=g_{1} \circ f_{1}$. If $G$ is a graph then $\Sigma(G)$ is the class of finite graphs which can be embedded in $G$.

Lemma A. Let $G$ be ultrahomogeneous and infinite. Then $\Sigma(G)$ has $A P$.

LEMMA B. Let $\Sigma$ be a class of finite graphs closed under isomorphism and substructure. Assume that $\Sigma$ has $A P$ and that $\Sigma$ contains arbitrarily large finite graphs. Then there is a countable ultrahomogeneous graph $G$ with $\Sigma(G)=\Sigma$.

LEMMA C. Let $G, H$ be countable ultrahomogeneous graphs, then $\Sigma(G)=\Sigma(H)$ iff $G \simeq H$.

The class $\Sigma(n)$ of finite graphs in which $K(n+1)$ cannot be embedded has AP and thus there is for each $n \geqslant 1$ a countable ultrahomogeneous graph $G(n)$ with $\Sigma(G(n))=\Sigma(n)$.

1. Reduction of the main theorem. Let $K(n)$ be the complete graph with $n$ vertices. If $G$ and $H$ are graphs let $G+H$ denote the graph obtained by taking the disjoint union of $G, H$, i.e., $G+H \cong\left\langle\left|G^{\prime}\right| \cup\left|H^{\prime}\right|, E_{G^{\prime}} \cup E_{H^{\prime}}\right\rangle$ where $G \cong G^{\prime}, H \cong H^{\prime}$ and $\left|G^{\prime}\right| \cap\left|H^{\prime}\right|=\varnothing$. If $G$ is a graph then the complementary graph $\tilde{G}$ is the graph $\left\langle|G|, E_{\tilde{G}}\right\rangle$ where $E_{\tilde{G}}=(|G| \times|G|) \backslash\left(E_{G} \cup\{(a, a): a \in|G|\}\right) . n G$ denotes the disjoint union of $n$ copies of $G$. Now let $E$ be the graph $K(2)+K(1)$. Let

$$
\mathscr{D}=\{K(n): 1 \leqslant n \in \omega\} \cup\{E, \tilde{E}\} \cup\{\tilde{K}(n): 1 \leqslant n \in \omega\} .
$$

Our main theorem can then be stated.

Theorem 1. Let $G_{1}, G_{2}$ be two countable ultrahomogeneous graphs such that for each $H \in \mathscr{D}, H$ can be embedded in $G_{1}$ just in case it can be embedded in $G_{2}$. Then $G_{1} \cong G_{2}$. Noticing that $K(m)$ can be embedded in $K(n)$, and $\tilde{K}(m)$ in $\tilde{K}(n)$ when $m<n$ we immediately have the following: 
COROLlARY. There are a countable number of countable ultrahomogeneous graphs.

If $G_{1}, G_{2}$ are such that $\Sigma\left(G_{1}\right) \cap \mathscr{D}=\Sigma\left(G_{2}\right) \cap \mathscr{D}$ and $E \notin \Sigma\left(G_{1}\right)$ or $\tilde{E} \notin \Sigma\left(G_{1}\right)$ it is not difficult to see that $G_{1} \cong G_{2}$. Let $m \leqslant \omega$ be the supremum of $\{n$ : $\left.K(n) \in \Sigma\left(G_{1}\right)\right\}$ and let $\tilde{m}$ be the supremum of $\left\{n: \tilde{K}(\tilde{n}) \in \Sigma\left(G_{1}\right)\right\}$. If $E \notin \Sigma\left(G_{1}\right)$ we have that $G_{1}, G_{2}$ are isomorphic to $m \tilde{K}(\tilde{m})$, and if $\tilde{E} \notin \Sigma\left(G_{1}\right)$ we see that $G_{1}, G_{2}$ are isomorphic to $\tilde{m} K(m)$. Thus we may assume that $E, \tilde{E}$ can both be embedded in $G_{1}$ and $G_{2}$. By an easy application of Ramsey's theorem we see that with $m, \tilde{m}$ defined as above at least one of $m$ and $\tilde{m}$ is $\omega$. Because $\tilde{G}_{1}, \tilde{G}_{2}$ are ultrahomogeneous whenever $G_{1}, G_{2}$ are, and $\Sigma\left(G_{1}\right) \cap \mathscr{D}=\Sigma\left(G_{2}\right) \cap \mathscr{D}$ iff $\Sigma\left(\tilde{G}_{1}\right) \cap \mathscr{D}=\Sigma\left(\tilde{G}_{2}\right) \cap \mathscr{D}$ there is no loss of generality in assuming that $\tilde{m}=\omega$. By Lemma $A \Sigma\left(G_{1}\right)$ and $\Sigma\left(G_{2}\right)$ have the amalgamation property. By Lemma $C$ it suffices to prove that $\Sigma\left(G_{1}\right)=\Sigma\left(G_{2}\right)$. This will follow easily from the next theorem, the proof of which occupies the remainder of the paper.

THEOREM 2'. Let $\Sigma$ be a class of finite graphs having the amalgamation property, and closed under isomorphism and subgraph. Assume that $E, \tilde{E}, K(m)$ and $\tilde{K}(n) \in \Sigma$ for each $1 \leqslant n<\omega$. Then every graph in which $K(m+1)$ cannot be embedded belongs to $\Sigma$.

Thus if $m=\omega, \Sigma\left(G_{1}\right)$ and $\Sigma\left(G_{2}\right)$ are the class of all finite graphs and if $m<\omega$ we have that $G_{1}, G_{2}$ are isomorphic to $G(m)$.

2. Derivations and Theorem 2. To prove Theorem $2^{\prime}$ we use the notion of a derivation.

Definition 1. Fix $m \geqslant 2$. We shall define what it means for an $n$-tuple of finite graphs $\left\langle G_{1}, \ldots, G_{n}\right\rangle$ to have a derivation from $\{E, \tilde{E}, K(m)\} \cup\{\tilde{K}(n): 1 \leqslant n<$ $\omega\}$. Let $\mathcal{G}_{0}(m)=\{\langle E\rangle,\langle\tilde{E}\rangle,\langle K(m)\rangle\} \cup\{\langle\tilde{K}(n)\rangle: 1 \leqslant n<\omega\}$ and if $\mathcal{G}_{r}(m)$ has been defined let $\mathcal{G}_{r+1}(m)$ be the class of finite sequences of finite graphs $\left\langle G_{1}, \ldots, G_{n}\right\rangle$ such that there are $\left\langle H_{1}, \ldots, H_{l}\right\rangle,\left\langle J_{1}, \ldots, J_{k}\right\rangle \in \mathcal{G}_{r}(m)$ with the property that for each $i, 1 \leqslant i \leqslant l$, and $j, 1 \leqslant j \leqslant k$, there is $A$, and there are embeddings $e_{0}: A \rightarrow H_{i}$ and $e_{1}: A \rightarrow J_{j}$ such that whenever $f_{0}: H_{i} \rightarrow C$ and $f_{1}$ : $J_{j} \rightarrow C$ are embeddings with $f_{0} \circ e_{0}=f_{1} \circ e_{1}$ there is an $s, 1 \leqslant s \leqslant n$, and an embedding $g: G_{s} \rightarrow C$.

A tuple of graphs $\left\langle G_{1}, \ldots, G_{n}\right\rangle$ is derivable from the given generating set if it belongs to $\mathcal{G}_{r}(m)$ for some $r<\omega$, and in this case we write $\vdash_{m} G_{1} \vee \ldots \vee G_{n}$. A graph $H$ is derivable if it belongs to the set $\mathcal{G}(m)=\left\{G:\langle G\rangle \in \cup_{r<\omega} \mathcal{G}_{r}(m)\right\}$, and we write $\vdash_{m} G$.

By induction on derivation we mean an induction on the hierarchy $\mathcal{G}_{r}(m)$.

Proposition. If $\Sigma$ has $A P$, is closed under isomorphism and subgraph and $E, \tilde{E}, K(m), \tilde{K}(n) \in \Sigma$ for $1 \leqslant n<\omega$ then $\mathcal{G}(m) \subset \Sigma$.

The proof of the Proposition is a trivial induction on derivation: we prove by induction on $r$ that if $\left\langle G_{1}, \ldots, G_{n}\right\rangle \in \mathcal{G}_{r}(m)$ then for some $j, 1 \leqslant j \leqslant n, G_{j} \in \Sigma$.

Clearly $\mathcal{G}(m) \subset \Sigma(m)$, since $\Sigma(m)$ has AP. To prove Theorem $2^{\prime}$ we shall establish: 
THEOREM 2. For $m \geqslant 2, \Sigma(m) \subset \mathcal{G}(m)$.

This proof is by induction on $m$, and is the subject of the remaining sections.

We complete this section with a digression showing that from quite general considerations it follows that $\mathcal{G}(m)$ is the class of all finite graphs which can be embedded in any ultrahomogeneous graph in which $E, \tilde{E}, K(m), \tilde{K}(n)$ can be embedded for $1<n<\omega$. Let $L$ be a first order language and let $\mathcal{C}$ be a class of structures for $L$ closed under isomorphism and substructures. $C$ is said to have the joint embedding property JEP if for each $A_{0}, A_{1} \in \mathcal{C}$ there is $B \in \mathcal{C}$ and there are embeddings $e_{0}: A_{0} \rightarrow B$ and $e_{1}: A_{1} \rightarrow B$. The definition of AP is just as it was for graphs. Below we find it convenient to regard joint embedding as merely amalgamation over an empty structure. Let $\mathcal{C}$ have AP and JEP and be such that whenever $A, B_{0}, B_{1} \in \mathcal{C}$ and $e_{0}, e_{1}$ are embeddings with $e_{0}: A \rightarrow B_{0}, e_{1}: A \rightarrow B_{1}$ there are $C_{1}, \ldots, C_{k} \in \mathcal{C}$ and embeddings $f_{0}^{i}, f_{1}^{i}, f_{0}^{i}: B_{0} \rightarrow C_{i}, f_{1}^{i}: B_{1} \rightarrow C_{i}$ with $f_{0}^{i} \circ e_{0}=f_{1}^{i} \circ e_{1}$ for $1 \leqslant i \leqslant k$ such that whenever $C \in \mathcal{C}$ and $f_{0}, f_{1}$ are embeddings $f_{0}: B_{0} \rightarrow C$ and $f_{1}: B_{1} \rightarrow C$ and $f_{0} \circ e_{0}=f_{1} \circ e_{1}$ there is an $i, 1<i<k$, and an embedding $f: C_{i} \rightarrow C$ with $f \circ f_{0}^{i}=f_{0}$ and $f \circ f_{1}^{i}=f_{1}$. We call $\left\langle C_{1}, \ldots, C_{k}\right\rangle$ an amalgamation sequence for $B_{0}, B_{1}$ over $A, e_{0}, e_{1}$.

When $\mathscr{D}$ is a subclass of $\mathcal{C}$ we may define what it means for a truple of members of $\mathcal{C}$ to be derivable from $\mathscr{D}$ in a manner analogous to that used for graphs. If $\left\langle C_{1}, \ldots, C_{n}\right\rangle$ is derivable from $\mathscr{D}$ we write $\mathscr{D} \vdash C_{1} \vee \ldots \vee C_{n}$ and we let $\mathscr{D}^{\prime}$ denote $\{C \in \mathcal{C}: \mathscr{D} \vdash C\}$. We then have the following extension of the Proposition.

LEMMA D. $\mathscr{D}^{\prime}$ is the intersection of all subclasses of $\mathcal{C}$ which contain $\mathscr{D}$, have $A P$, $J E P$ and are closed under isomorphism and substructure.

Proof. That $\mathscr{D}^{\prime}$ is a subclass of any subclass of $\mathcal{C}$ with AP, JEP and closed under isomorphism and substructures is clear by induction on derivation as before.

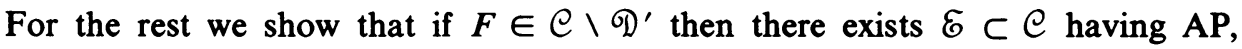
JEP, closed under isomorphism and substructures such that $F \notin \mathcal{E}$.

Let $C_{0}$ be a set containing at least one member of each isomorphism type of $C$, and without loss take $\mathscr{D} \subset C_{0}$.

Let $I$ be the smallest set containing $\{\{D\}: D \in \mathscr{D}\}$ and such that if $\gamma, \delta \in I$, $A \in C_{0}$, and $e_{0}, e_{1}$ are embeddings of $A$ into members of $\bigodot_{0}$ then $\left\langle\gamma, \delta, A, e_{0}\right.$, $\left.e_{1}\right\rangle \in I$. $I$ is an index set for the construction of $\delta$. Fix a well ordering < of $I$ such that $\gamma, \delta$ are less than $\left\langle\gamma, \delta, A, e_{0}, e_{1}\right\rangle$. Next choose by recursion a mapping $c$ : $I \rightarrow \mathrm{C}_{0}$ such that

(i) $c(\{D\})=D$ for $D \in \mathscr{D}$,

(ii) $F$ is not derivable from $\mathscr{D} \cup\{c(\gamma): \gamma<\iota\}$ for $\iota \in I$, and

(iii) if $\iota=\left\langle\gamma, \delta, A, e_{0}, e_{1}\right\rangle, c(\gamma)=B_{0}, c(\delta)=B_{1}$ and $e_{0}: A \rightarrow B_{0}$ and $e_{1}: A \rightarrow B_{1}$ are embeddings then $\iota$ is the index of a member of $C_{0}$ which amalgamates $B_{0}, B_{1}$ over $A, e_{0}, e_{1}$.

The recursion is possible provided that if $D_{0}, D_{1} \in \mathscr{D}, A \in \mathcal{C}$ and $e_{0}: A \rightarrow D_{0}$, $e_{1}: A \rightarrow D_{1}$ are embeddings then there are $C \in \mathcal{C}$ and embeddings $f_{1}: D_{0} \rightarrow C, f_{1}$ : $D_{1} \rightarrow C$ such that $f_{0}^{\circ} e_{0}=f_{1} \circ e_{1}$ and $F$ cannot be derived from $\mathscr{D} \cup\{C\}$. 
Let $\left\langle C_{1}, \ldots, C_{k}\right\rangle$ be an amalgamation sequence for $D_{0}, D_{1}$ over $A, e_{0}, e_{1}$. Now D) $\vdash C_{1} \vee \ldots \vee C_{k}$.

The following claim gives the result.

Claim. If $\mathscr{D} \vdash B_{1} \vee \ldots \vee B_{n}$ and $\mathscr{D} \cup\left\{B_{1}\right\} \vdash G_{1} \vee \ldots \vee G_{k}$. Then $\mathscr{D} \vdash G_{1}$ $\vee \ldots \vee G_{k} \vee B_{2} \vee \ldots \vee B_{n}$.

This claim is easily proved by induction on the derivation of $\left\langle G_{1}, \ldots, G_{k}\right\rangle$ from OD $\cup\left\{B_{1}\right\}$.

Now if $\mathscr{D} \cup\left\{C_{i}\right\} \vdash F$ for $1 \leqslant i \leqslant k$ then since $\mathscr{D} \vdash C_{1} \vee \ldots \vee C_{k}$ we have by $k$ applications of the claim that $\mathscr{D} \vdash F$, a contradiction. Taking $\mathcal{E}$ to be the class of all $C \in \mathcal{C}$ embeddable in some $C(\iota), \iota \in I$, the proof of Lemma $\mathrm{D}$ is complete.

In the case of graphs with $\mathscr{D}=\{E, \tilde{E}, K(m)\} \cup\{\tilde{K}(n): 1<n<\omega\}$ any class including $\mathscr{D}$ and having AP automatically has JEP. From Lemmas A, B, a class of finite graphs including $\mathscr{D}$, closed under isomorphism, substructures, and having AP is necessarily the class of all finite graphs embeddable in some countable ultrahomogeneous graph. Thus as claimed at the beginning of the digression from Lemma $\mathrm{D}, \mathcal{G}(\mathrm{m})$ is the class of all finite graphs embeddable in every ultrahomogeneous graph in which all members of $\mathscr{D}$ are embeddable.

3. Outline of the proof. In this section we list six lemmas from which Theorem 2 will follow. To state these we first require two definitions.

Definition 2. Let $G, H$ be graphs, $a \in|G|, b \in|H|$. Then $[(G, a),(H, b)]$ is the graph obtained from $G+H$ by identifying the copies of $a, b$. Thus if $G, H$ are copies of $K_{2}, a \in|G|$ and $b \in|H|$ then $[(G, a),(H, b)]$ is isomorphic to $\tilde{E}$.

Definition 3. Let $G, H$ be graphs. For each $a \in|G|$ let $H_{a}$ be the graph with universe $\{(a, b): b \in|H|\}$ and with an edge between $\left(a, b_{1}\right)$ and $\left(a, b_{2}\right)$ iff $\left(b_{1}, b_{2}\right) \in E_{H}$. The graph $G \otimes H$ is then the graph with universe $|G| \cup$ $\cup_{a \in|G|}\left|H_{a}\right|$ and with

$$
\begin{gathered}
E_{G \otimes H}=E_{G} \cup \bigcup_{a \in|G|} E_{H_{a}} \cup\{(a,(a, b)): b \in|H|, a \in|G|\} \\
\cup\{((a, b), a): b \in|H|, a \in|G|\} .
\end{gathered}
$$

That is, a copy of $H$ is suspended from each vertex of $G$, and there is an edge between $a \in|G|$, and each vertex of the copy $H_{a}$ suspended from $a$.

Definition 4. Let $n \geqslant 2$. Let $\mathcal{G}^{*}(m)$ be the class of all graphs $H$ such that for every $G \in \mathcal{G}(m), a \in|G|, b \in|H|$ the graph $[(G, a),(H, b)] \in \mathcal{G}(m)$.

Taking $G=K(1)$ we see at once that $\mathcal{G}^{*}(m) \subset \mathcal{G}(m)$. As will be made clear below the whole trick is to show that in fact $\Sigma(m) \subset \mathcal{G}^{*}(m)$.

Let $L(n)$ denote a line with $(n-1)$ edges and $n$ vertices. Thus $\tilde{E} \cong L(3)$.

Theorem 2 is proved by induction, and thus we assume that if $2<m^{\prime}<m$ then $\Sigma\left(m^{\prime}\right) \subset \mathcal{G}\left(m^{\prime}\right)$. The plan of the proof is as follows:

LEMMA 1. $K(m)+L(3), K(m)+K(m)$ and $L(3)+L(3)$ all belong to $\mathcal{G}(m)$.

LEMMA 2. $\mathcal{G}(m)$ is closed under + , indeed $\vdash_{m} G_{1} \vee \ldots \vee G_{n}$ and $\vdash_{m} H$ imply $\vdash_{m}\left(G_{1}+H\right) \vee \ldots \vee\left(G_{n}+H\right)$. 
Lemma 3. $\vdash_{m} K(m) \otimes K(m-1)$ and if $m \geqslant 3, \vdash_{m} K(m) \otimes L(3)$. Also $\vdash_{m} L(3) \otimes$ $K(m-1)$ and if $m \geqslant 3$ then $\vdash_{m} L(3) \otimes L(3)$.

Lemma 4. Let $\vdash_{m} G$. Then $\vdash_{m} G \otimes K(m-1)$ and if $m \geqslant 3$ then $\vdash_{m} G \otimes L(3)$.

Remark 1. From Lemma 4 it follows immediately that $K(m) \in G^{*}(m)$. In fact it is not hard to see that this statement is equivalent to $G \otimes K(m-1) \in \mathcal{G}(m)$. If $K(m) \in G^{*}(m)$ then $K(2) \in G^{*}(m)$ and it is easy to see that $K(2) \in G^{*}(m) \Rightarrow L(n)$ $\in G^{*}(m)$ for all $n$.

Lemma 5. Let $\vdash_{m} G, a \in|G|$, and $H \in \Sigma(m-1)$. Then $\vdash_{m}[(G, a),(\{h\} \otimes H, h)]$, where $\{h\}$ denotes the graph with sole vertex $h$.

Lemma 6. If $K(m+1)$ is not embeddable in $H$ then $H \in G^{*}(m)$, i.e. $\Sigma(m) \subset$ $G^{*}(m)$.

Although the lemmas are presented in their logical order we shall prove them in the order: $6,2,4,5,1,3$. This departure from the order above is made for two reasons. First, Lemma 6 contains the key idea, and the other lemmas are merely used to set it up. Next Lemmas 2, 4, and 5 are relatively easy to prove and the proofs of Lemmas 2 and 4 appear in modified form in the proofs of Lemmas 1 and 3 respectively. Lemmas 1 and 3 are quite long and complicated. Thus we hope the order chosen is optimal from the point of view of a reader who wishes to see first the heart of the argument and then the necessary underpinnings in order of complexity.

In $\S 4$ we shall prove Lemmas 6, 2, 4 and 5. Lemma 1 will be proved in $\S 5$ and Lemma 3 in $\$ 6$. Many of the arguments turn on the possibilities for amalgamation of two graphs $B_{0}, B_{1}$ over their intersection $A$. These are best represented pictorially. A typical picture is shown in Figure 0.
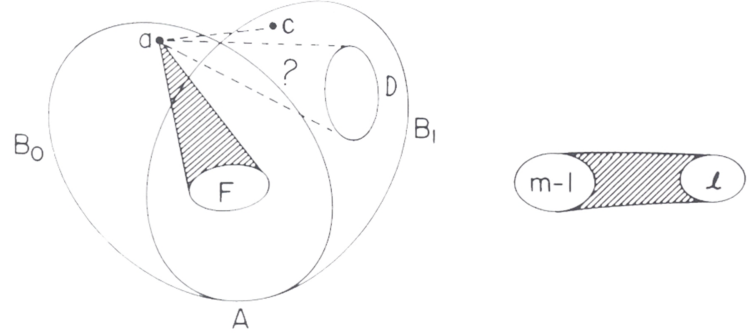

FigURE 0

The amalgamation is such that no points in $B_{0} \backslash A$ and $B_{1} \backslash A$ can be identified in any amalgamation. The dotted line from $a$ to $c$ indicates that it is not known (a priori) whether there can be an edge joining $a$ and $c$, in a given amalgamation $C$, and the cone from $a$ to $D$ indicates that $D$ is a subgraph of $B_{1}$ lying in $B_{1} \backslash A$, and some, possibly none, possibly all, edges from $a$ to vertices in $D$ may occur in a given amalgamation. This may happen in two ways. In one the edges may depend 
on the given amalgamation. In the second we may know what edges occur but have no convenient way to represent them pictorially. The cone from $a$ to $F$ indicates that there is an edge between each vertex of $F$ and $a$, i.e., $\{a\} \cup|F|$ determines a subgraph isomorphic to $\{a\} \otimes F$. A circled number $m$ indicates a copy of $K(m)$. The cone from $K(m-1)$ to $K(l)$ indicates that every edge is present, i.e. we have $K(m-1+l)$. The reader should continue to bear in mind the following: $E$ is $K(2)+K(1), \tilde{E}$ is $L(3)$ and $\tilde{K}(n)$ is $n K(1)$.

4. The key idea and the easy supporting lemmas. The reader will find the crux of the whole paper in the following:

LeMMA 6. $\Sigma(m) \subset \mathcal{G} *(m)$.

Proof. Let $H$ be a graph in which $K(m+1)$ cannot be embedded. Let $G \in$ $\mathcal{G}(m), a \in|G|$ and $b \in|H|$. We want to show that

$$
\vdash_{m}[(G, a),(H, b)] \text {. }
$$

We proceed by induction on $|H|$, i.e. the cardinality of $H$. Let $H^{-}$denote the graph obtained from $H$ by deleting $b$. There are three cases.

Case 1 . The vertex $b$ is not joined to any other vertex of $H$. Clearly

$$
[(G, a),(H, b)] \cong G+H^{-} .
$$

Since $\left|H^{-}\right|<|H|, H^{-} \in G^{*}(m)$ whence $\vdash_{m} H^{-}$. From Lemma 2, $\vdash_{m} G+H^{-}$.

Case 2. The vertex $b$ is joined to every vertex of $H$. Then $K(m)$ cannot be embedded in $H^{-}$since $K(m+1)$ is not embeddable in $H$. Taking $H$ in Lemma 5 to be $H^{-}$we immediately have $(+)$as required.

Case 3. There are vertices $b_{0}, b_{1}$ of $H$ distinct from $b$ such that $b$ is joined to $b_{0}$ but not to $b_{1}$. Let $H_{i}$ be isomorphic to the restriction of $H$ to $|H| \backslash\left\{b, b_{i}\right\}$ and let $|G|,\left|H_{0}\right|,\left|H_{1}\right|,\{b\}$ be pairwise disjoint. Let $b^{\prime} \notin|G| \cup\left|H_{0}\right| \cup\left|H_{1}\right| \cup\{b\}$. Now we form the graph $\mathrm{H}^{+}$as follows:

(i) $\left|H^{+}\right|=\left|H_{0}\right| \cup\left|H_{1}\right| \cup\left\{b, b^{\prime}\right\}$;

(ii) the restriction of $H^{+}$to $\left|H_{0}\right|,\left|H_{1}\right|$ are $H_{0}, H_{1}$ respectively;

(iii) there are no edges between $\left|H_{0}\right|,\left|H_{1}\right| ; b, b^{\prime}$ are not joined;

(iv) there is an isomorphism from $H$ onto the restriction of $H^{+}$to $\left|H_{0}\right| \cup\left\{b, b^{\prime}\right\}$ which extends the isomorphism of $H \backslash\left\{b, b_{0}\right\}$ onto $H_{0}$, fixes $b$ and takes $b_{1}$ to $b^{\prime}$; and

(v) there is an isomorphism from $H$ with the edge between $b$ and $b_{0}$ deleted onto the restriction of $H^{+}$to $\left|H_{1}\right| \cup\left\{b, b^{\prime}\right\}$ which extends the isomorphism of $H \backslash\left\{b, b_{1}\right\}$, fixes $b$ and takes $b_{0}$ onto $b^{\prime}$.

We repeat that $H^{+}$has the property that restricting to $\left|H_{1}\right| \cup\left\{b, b^{\prime}\right\}$ and adding an edge between $\left\{b, b^{\prime}\right\}$ gives a copy of $H$, and restriction to $\left|H_{0}\right| \cup\left\{b, b^{\prime}\right\}$ gives a copy of $H$. In each case the isomorphism fixes $b . H^{+}$is depicted in Figure 1. The question marks emphasize that some edges may be present and some not between $b, b^{\prime}$ and the rest of the graph. 


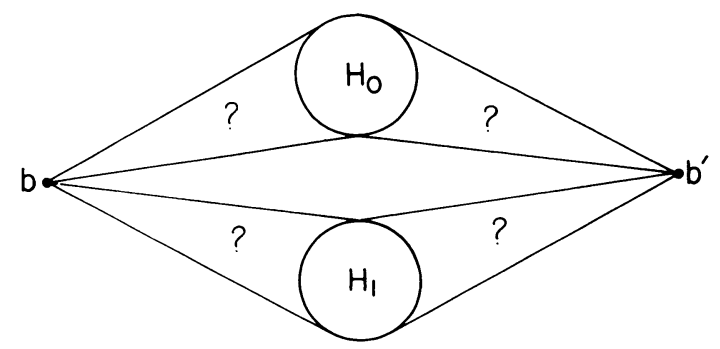

FIGURE 1

Let $H_{0}, H_{1}$ be the restrictions of $H^{+}$to $\left|H_{0}\right| \cup\{b\},\left|H_{1}\right| \cup\{b\}$ respectively; and let $H_{0}^{\prime}, H_{1}^{\prime}$ be the restrictions of $H^{+}$to $\left|H_{0}\right| \cup\left\{b^{\prime}\right\},\left|H_{1}\right| \cup\left\{b^{\prime}\right\}$, respectively.

By the induction hypothesis $H_{0}^{*}, H_{1}, H_{0}^{\prime}, H_{1}^{\prime}$ all belong to $\mathcal{G}^{*}(m)$. Now let $G^{+}=\left[(G, a),\left(H^{+}, b\right)\right]$. Deleting the vertex $b$ from $G^{+}$we obtain the graph $G^{-}+\left[\left(H_{0}^{\prime}, b^{\prime}\right),\left(H_{1}^{\prime}, b^{\prime}\right)\right]$ where $G^{-}$is obtained from $G$ by deleting $a$. Now - $_{m} G^{-}$ since $\vdash_{m} G$ and $\vdash_{m}\left[\left(H_{0}^{\prime}, b^{\prime}\right),\left(H_{1}^{\prime}, b^{\prime}\right)\right]$ since $H_{0}^{\prime}, H_{1}^{\prime} \in \mathcal{G}^{*}(m)$ whence $\vdash_{m} G^{-}+$ $\left[\left(H_{0}^{\prime}, b^{\prime}\right),\left(H_{1}^{\prime}, b^{\prime}\right)\right]$ by Lemma 2 . Deleting the vertex $b^{\prime}$ from $G^{+}$we obtain the graph

$$
\left[\left(\left[(G, a),\left(H_{0}^{*}, b\right)\right], a\right),\left(H_{1}, b\right)\right] .
$$

Since $H_{0} \in \mathcal{G}^{*}(m)$ we have $\vdash_{m}\left[(G, a),\left(H_{0}^{*}, b\right)\right]$. Next since $H_{1} \in \mathcal{G}^{*}(m)$ we have

$$
\vdash_{m}\left[\left(\left[(G, a),\left(H_{0}^{*}, b\right)\right], a\right),\left(H_{1}^{*}, b\right)\right] \text {. }
$$

Thus deleting either $b$ or $b^{\prime}$ from $G^{+}$gives us a graph in $\mathcal{G}(m)$. We can amalgamate over a common subset because then the edges on the subset are fixed.

Consider now the amalgamation of $G^{+} \uparrow\left(\left|G^{+}\right| \backslash\left\{b^{\prime}\right\}\right)$ and $G^{+} \uparrow$ $\left(\mid G^{+} \wedge\{b\}\right)$ over $\mid G^{+} \backslash\left\{b, b^{\prime}\right\}$ by embeddings $g_{0}, g_{1}$ respectively into a finite graph $K$. Now $g_{0}(b) \neq g_{1}\left(b^{\prime}\right)$ since $b$ is connected to the image of $b_{0}$ in $H_{1}$ but not to that of $b_{1}$ in $H_{0}$. However $b^{\prime}$ is either connected to both or neither of these vertices according as there is an edge from $b_{0}$ to $b_{1}$. Thus $K \uparrow\left(\operatorname{Im} g_{0} \cup \operatorname{Im} g_{1}\right)$ is either isomorphic to $G^{+}$or the graph $G^{++}$where $G^{++}$is obtained from $G^{+}$by adding an edge between $b$ and $b^{\prime}$. Now we are done because the graph $[(G, a),(H, b)]$ is embeddable in both $G^{+}$and $G^{++}$; in both cases the embedding is the identity on $G$; in the first case $b_{1}$ is mapped into $b^{\prime}$ and the embedding extends the isomorphism of $H \backslash\left\{b, b_{1}\right\}$ and $H_{1}$ and in the second case $b_{0}$ is mapped into $b^{\prime}$ and the embedding extends the isomorphism of $H \backslash\left\{b, b_{0}\right\}$ and $H_{0}$.

This completes the proof of Lemma 6.

We now turn to Lemma 2. $\mathcal{G}(m)$ is closed under + . Indeed $\vdash_{m} G_{1} \vee \ldots \vee G_{n}$ and $\vdash_{m} H$ imply $\vdash_{m}\left(G_{1}+H\right) \vee \ldots \vee\left(G_{n}+H\right)$.

Proof. From Lemma 1 it is immediate that $K(m)+K(1), L(3)+K(1), K(2)+$ $K(1)+K(1)$ and $n K(1)+K(1)$ are all in $\mathcal{G}(m)$. Note that $K(2)+K(1)+K(1)$ is a subgraph of $L(3)+L(3)$. By induction on derivation we show that $\mathcal{G}(m)$ is closed under the addition of $K(1)$. Specifically we prove that $\vdash_{m}\left\langle G_{1}, \ldots, G_{n}\right\rangle$ implies $\vdash_{m}\left\langle G_{1}+K(1), \ldots, G_{n}+K(1)\right\rangle$. This is true by the above for the members of 
$\mathcal{G}_{0}(m)$. So assume that $\vdash_{m}\left\langle H_{1}+K(1), \ldots, H_{l}+K(1)\right\rangle$ and $\vdash_{m}\left\langle J_{1}+K(1), \ldots, J_{k}\right.$ $+K(1)>$ and for $1 \leqslant i \leqslant l$ and $1 \leqslant j \leqslant k$ there are $A$ and embeddings $e_{0}: A \rightarrow H_{i}$ and $e_{1}: A \rightarrow J_{j}$ such that whenever $K$ and embeddings $f_{0}: H_{i} \rightarrow K, f_{1}: J_{j} \rightarrow K$ have $f_{0} \circ e_{0}=f_{1} \circ e_{1}$ there are $q, 1 \leqslant q \leqslant n$, and an embedding $f: G_{q} \rightarrow K$. Let $1<i<l$, $1<j<k$ and fix $A, e_{0}, e_{1}$ as above. Now $e_{0}, e_{1}$ can be extended uniquely to embeddings $\hat{e}_{0}, \hat{e}_{1}$ of $A+K(1)$ into $H_{i}+K(1)$ and $J_{j}+K(1)$ respectively. Now let $\hat{f}_{0}, \hat{f}_{1}$ be embeddings of $H_{i}+K(1)$ and $J_{j}+K(1)$ respectively into $\hat{K}$ with $\hat{f}_{0} \circ \hat{e}_{0}=$ $\hat{f}_{1} \circ \hat{e}_{1}$, where $|\hat{K}|=\operatorname{Im} \hat{f}_{0} \cup \operatorname{Im} \hat{f}_{1}$. Then $f_{0}=\hat{f}_{0} \uparrow H_{i}$ and $f_{1}=\hat{f}_{1} \uparrow J_{j}$ are embeddings into $K$, the subgraph of $\hat{K}$ resulting from deletion of the image of $K(1)$. But then the embedding $g: G_{q} \rightarrow K$ may be extended to $\hat{g}: G_{q}+K(1) \rightarrow \hat{K}$ in the obvious way.

It follows in particular that $n K(1)+K(m), n K(1)+L(3), n K(1)+K(2)+K(1)$ are all in $\mathcal{G}(m)$.

An induction like the above establishes the following sublemma which is also used in the proof of Lemma 1.

Sublemma 2. If $\{K(m)+H, L(3)+H, K(2)+K(1)+H, n K(1)+H\} \subseteq \mathcal{G}(m)$ and $\vdash_{m} G_{1} \vee \ldots \vee G_{n}$, then

$$
\vdash_{m}\left(G_{1}+H\right) \vee \ldots \vee\left(G_{n}+H\right)
$$

Applying Lemma 1 and the above $K(m)+K(m), L(3)+K(m), K(2)+K(1)+$ $K(m)$ and $n K(1)+K(m)$ are all in $\mathcal{G}(m)$. Thus by a similar induction $\mathcal{G}(m)$ is closed under addition of $K(m)$. Similarly $\mathcal{G}(m)$ is closed under addition of $L(3)$. Now let $t_{m} H$. We see that $H+K(m), H+L(3), H+(2)+K(1)$ and $H+n K(1)$ all belong to $\mathcal{S}(m)$, whence $\mathcal{G}(m)$ is closed under addition of $H$ by the same type of induction, that is $\vdash_{m} G_{1} \vee \ldots \vee G_{n}$ implies $\vdash_{m}\left(G_{1}+H\right) \vee \ldots \vee\left(G_{n}+H\right)$, and the lemma follows.

LEMMA 4. Let $\vdash_{m} G . \vdash_{m} G \otimes K(m-1)$ and if $m \geqslant 3$ then $\vdash_{m} G \otimes L(3)$.

Proof. Again we use induction on the derivation of $G$. First we check the starting cases. By Lemma 3

$$
K(m) \otimes K(m-1), \quad L(3) \otimes K(m-1) \in \mathcal{G}(m) .
$$

Also $\vdash_{m}(K(2)+K(1)) \otimes K(m-1)$ since this graph is $(K(2) \otimes K(m-1))+K(m)$ and $\mathcal{G}(m)$ is closed under + . Finally $\vdash_{m}(n K(1)) \otimes K(m-1)$ since this is just $\vdash_{m} n K(m)$. Similarly with $L(3)$ in place of $K(m-1)$ when $m>3$.

For the induction step assume that $r_{m}\left\langle H_{1} \otimes K(m-1), \ldots, H_{k} \otimes K(m-1)\right\rangle$ and $r_{m}\left\langle J_{1} \otimes K(m-1), \ldots, J_{l} \otimes K(m-1)\right\rangle$ and that $\left\langle G_{1}, \ldots, G_{n}\right\rangle$ is such that for $1<i<k$ and $1 \leqslant j \leqslant l$ there are $A$, and embeddings $e_{0}: A \rightarrow H_{i}$ and $e_{1}$ : $A \rightarrow J_{j}$ such that for any $K$ and embeddings $f_{0}: H_{i} \rightarrow K$ and $f_{1}: J_{j} \rightarrow K$ with $f_{0} \circ e_{0}=f_{1} \circ e_{1}$ there exists $1 \leqslant q \leqslant n$ and an embedding $g: G_{q} \rightarrow K$.

Let $n_{1}$ be the maximum of $\left\{\left|H_{i}\right|+\left|J_{j}\right|: 1 \leqslant i<k, 1<j<l\right\}$. By Lemma $2, \vdash_{m} G$ and $\vdash_{m} J_{1} \vee \ldots \vee J_{k}$ imply $\vdash_{m}\left(J_{1}+G\right) \vee \ldots \vee\left(J_{k}+G\right)$. Since $\vdash_{m} n_{1} K(m-1)$ it follows that

$$
\begin{aligned}
\vdash_{m}\left\langle H_{1} \otimes K(m-1)+\left(n_{1}-\mid\right.\right. & \left.H_{1} \mid\right) K(m-1), \ldots, \\
& \left.H_{k} \otimes K(m-1)+\left(n_{1}-\left|H_{k}\right|\right) K(m-1)\right\rangle .
\end{aligned}
$$


The same result with $H$ replaced by $J$ and $k$ by $l$ also follows.

Now let $1 \leqslant i \leqslant k, 1 \leqslant j \leqslant l$ and fix $A, e_{0}: A \rightarrow H_{i}$ and $e_{1}: A \rightarrow J_{j}$ as above. Let $\hat{A}$ be $A \otimes K(m-1)+\left(n_{1}-|A|\right) K(m-1)$. Let $\hat{e}_{0}$, $\hat{e}_{1}$ be embeddings of $\hat{A}$ into $\hat{H}_{i}=H_{i} \otimes K(m-1)+\left(n_{1}-\left|H_{i}\right|\right) K(m-1)$ and $\hat{J}_{j}=J_{j} \otimes K(m-1)+$ $\left(n_{1}-\left|J_{j}\right|\right) K(m-1)$ extending $e_{0}, e_{1}$ respectively such that:

(i) no vertex of $\hat{A} \backslash A$ is mapped into $H_{i}$ or $J_{j}$ where $A, H_{i}, J_{j}$ are viewed in the natural way as subsets of $\hat{A}, \hat{H}_{i}, \hat{J}_{j}$ respectively. Thus the additional copies of $K(m-1)$ are carried onto additional copies of $K(m-1)$; and

(ii) if an edge occurs between a vertex of $H_{i} \backslash e_{0}(A)$ and a vertex of the image under $\hat{e}_{0}$ of some one of the $\left(n_{1}-|A|\right)$ copies of $K(m-1)$ then no edge occurs between a vertex of $J_{j}$ and the image of that copy of $K(m-1)$ under $\hat{e}_{1}$; and similarly with $H_{i}, J_{j}$ and $\hat{e}_{0}, \hat{e}_{1}$ interchanged.

Now if $\hat{f}_{0}, \hat{f}_{1}$ are embeddings of $\hat{H}_{i}$ and $\hat{J}_{j}$ respectively into $\hat{L}$ with $\hat{f}_{0} \circ \hat{e}_{0}=\hat{f}_{1} \circ \hat{e}_{1}$ and $|\hat{L}|=\operatorname{Im} \hat{f}_{0} \cup \operatorname{Im} \hat{f}_{1}$, then $f_{0}=\hat{f}_{0} \uparrow H_{i}$ and $f_{1}=\hat{f}_{1} \uparrow J_{j}$ are embeddings such that $f_{0} \circ e_{0}=f_{1} \circ e_{1}$. Let $L=\hat{L} \uparrow\left(\operatorname{Im} f_{0} \cup \operatorname{Im} f_{1}\right), 1 \leqslant q \leqslant n$, and $g: G_{q} \rightarrow L$ be an embedding. Then $g$ can be extended to an embedding $\hat{g}: G_{g} \otimes K(m-1) \rightarrow \hat{L}$. A similar proof works with $L(3)$ in place of $K(m-1)$ when $m \geqslant 3$. This completes the proof of Lemma 4.

LEMMA 5. Let $\vdash_{m} G, a \in|G|$ and $H \in \Sigma(m-1)$. Then $\vdash_{m}[(G, a),(\{h\} \otimes H, h)]$ where $\{h\}$ denotes the graph with sole vertex $h$.

Proof. For any graph $F$ let $\{h\} \otimes F$ be denoted $F^{\prime}$.

$K(m)$ is not embeddable in $H$ whence by the induction hypothesis when $m \geqslant 3$, $\vdash_{m-1} H$, and, by inspection, when $m=2, H$ is isomorphic to $n K(1)$ for some $n$.

Notice that Lemma 5 holds for $H=K(1)$ because in this case $\left[(G, a),\left(H^{\prime}, h\right)\right]$ is embeddable in $G \otimes K(m-1)$ which belongs to $\mathcal{G}(m)$ by Lemma 4 . By iteration Lemma 5 holds for $H=n K(1)$ because

$$
\begin{aligned}
& {\left[(G, a),\left((n K(1))^{\prime}, h\right)\right]} \\
& \quad \cong\left[\left(\ldots\left(\left[\left(\left[(G, a),\left((K(1))^{\prime}, h\right)\right], a\right),\left((K(1))^{\prime}, h\right)\right], a\right), \ldots, a\right),\left((K(1))^{\prime}, h\right)\right] .
\end{aligned}
$$

When $m=2$ this is enough. Now suppose $m>2$. From Lemma $4, G \otimes K(m-1)$, $G \otimes L(3)$ are both members of $\mathcal{G}(m)$, whence Lemma 5 holds for $H=K(m-1)$ and $H=L(3)$. Since $K(2)$ is embeddable in $K(m-1)$ Lemma 5 holds for $H=$ $K(2)$. Since Lemma 5 holds for $K(2)$ and $K(1)$ it also holds for $H=K(2)+K(1)$ because

$$
\left[(G, a),\left((K(2)+K(1))^{\prime}, h\right)\right] \cong\left[\left(\left[(G, a),\left((K(2))^{\prime}, h\right)\right], a\right),\left((K(1))^{\prime}, h\right)\right] .
$$

Now we complete the proof of Lemma 5 by induction on the derivation of $H$ in $\mathcal{G}(m-1)$. We show that if $\left\langle H_{1}, \ldots, H_{n}\right\rangle \in \mathcal{G}_{r}(m-1)$ then $\langle[(G, a)$, $\left.\left.\left(H_{1}^{\prime}, h\right)\right], \ldots,\left[(G, a),\left(H_{n}^{\prime}, h\right)\right]\right\rangle \in \mathcal{G}_{s}(m)$ for some $s$. From above this is true for $r=0$. Now assume that

$$
\begin{aligned}
\left\langle\left[(G, a),\left(I_{1}^{\prime}, h\right)\right], \ldots,\right. & {\left.\left[(G, a),\left(I_{k}^{\prime}, h\right)\right]\right\rangle, } \\
\langle & {\left.\left[(G, a),\left(J_{1}^{\prime}, h\right)\right], \ldots,\left[(G, a),\left(J_{l}^{\prime}, h\right)\right]\right\rangle \in \mathcal{G}_{s}(m) }
\end{aligned}
$$


and that for $1 \leqslant i \leqslant k, 1 \leqslant j \leqslant l$ there are $A$ and embeddings $e_{0}: A \rightarrow I_{i}, e_{1}$ : $A \rightarrow J_{j}$ such that whenever $f_{0}: I_{i} \rightarrow K, f_{1}: J_{j} \rightarrow K$ are embeddings with $f_{0} \circ e_{0}=$ $f_{1} \circ e_{1}$ there are $1 \leqslant q \leqslant n$ and an embedding $g: H_{q} \rightarrow K$.

Fix $1 \leqslant i \leqslant k, 1 \leqslant j \leqslant l, A, e_{0}, e_{1}$ as above. Let $\hat{e}_{0}, \hat{e}_{1}$ be the embeddings of $\left[(G, a),\left(A^{\prime}, h\right)\right]$ into $\left[(G, a),\left(I_{i}^{\prime}, h\right)\right]$ and $\left[(G, a),\left(J_{j}^{\prime}, h\right)\right]$ respectively which extend $e_{0}$, $e_{1}$ respectively and are the identity on $G$. Now let $\hat{f}_{0}, \hat{f}_{1}$ be embeddings of $[(G, a)$, $\left.\left(I_{i}^{\prime}, h\right)\right]$ and $\left[(G, a),\left(J_{j}^{\prime}, h\right)\right]$ respectively into $\hat{K}$ with $|\hat{K}|=\operatorname{Im} \hat{f}_{0} \cup \operatorname{Im} \hat{f}_{1}$ and $\hat{f}_{0} \circ \hat{e}_{0}=\hat{f}_{1} \circ \hat{e}_{1}$. Then $f_{0}=\hat{f}_{0} \uparrow\left|I_{i}\right|$ and $f_{1}=\hat{f}_{1} \uparrow\left|J_{j}\right|$ are embeddings satisfying $f_{0} \circ e_{0}=f_{1} \circ e_{1}$. Let $K=\hat{K} \uparrow\left(\operatorname{Im} f_{0} \cup \operatorname{Im} f_{0}\right)$ and $1<q \leqslant n$ and $g: H_{q} \rightarrow K$ be an embedding. There is a unique extension of $g$ to $\hat{g}$, embedding $\left[(G, a),\left(H_{q}^{\prime}, h\right)\right]$ in $\hat{K}$ with $\hat{g}$ the identity on $G$.

This completes the proof of Lemma 5.

5. Proof of Lemma 1. In this section we prove

LEMMA 1. $K(m)+L(3), K(m)+K(m)$ and $L(3)+L(3)$ all belong to $\mathcal{G}(m)$.

The proof of the lemma is broken into two main parts. First we prove the lemma for $m=2$ and then consider the proof when $m>2$.

5.1. Let $m=2$. We prove

(i) $\vdash_{2} K(2)+K(1)+K(1)$;

(ii) $\vdash_{2} L(3)+K(1)$;

whence by Sublemma $2, \mathcal{G}(2)$ is closed under addition of $K(1)$.

(iii) $\vdash_{2} K(2)+K(2)$;

(iv) $\vdash_{2} K(2)+L(3)$.

Thus by Sublemma $2, \mathcal{G}(2)$ is closed under addition of $K(2)$.

(v) $\vdash_{2} L(3)+L(3)$.

We thank Ron Morrow for proofs of (i) and (iv) and for the simpler argument for (v) which we reproduce. Let $S(n)$ denote $\{a\} \otimes n K(1)$, a star with $n$ rays, and let $C(n)$ denote the cycle of length $n$.

(i) From the amalgamation of $K(2)+K(1)$ and $4 K(1)$ over $2 K(1)$ we obtain

$$
\vdash_{2}\langle K(2)+2 K(1), S(3)+K(1)\rangle
$$

see Figure 2(1). Thus $t_{2}\langle K(2)+2 K(1), L(3)+K(1)\rangle$. From this and the amalgamation of two copies of $L(3)+K(1)$ over $K(2)+K(1)$ we obtain

$$
\vdash_{2}\langle K(2)+2 K(1), C(4)+K(1)\rangle \text {, }
$$

see Figure 2(2). From the amalgamation of two copies of $K(2)+K(1)$ over $2 K(1)$ shown in Figure 2(3) we see that

$$
\vdash_{2}\langle K(2)+K(2), L(4)\rangle .
$$

From (1), (3) and the amalgamation shown in Figure 2(4) of $2 K(2)$ and $L(3)+$ $K(1)$ over $K(2)+K(1)$ we have

$$
\vdash_{2}\langle K(2)+2 K(1), L(4)\rangle \text {. }
$$

We have shown the amalgamation for the only nontrivial pairing in the derivation. Now from (1), (4) and the amalgamation shown in Figure 2(5) of $L(4)$ and $S(3)$ 
over $L(3)$, we obtain

$$
\vdash_{2}\langle K(2)+2 K(1),[(C(4), a),(K(2), b)]\rangle
$$

where $a, b$ are vertices of $C(4), K(2)$ respectively. Finally from (2), (5) and the amalgamation of Figure 2(6) of $[(C(4), a),(L(2), b)]$ and $C(4)+K(1)$ over $C(4)$ we obtain $t_{2} K(2)+2 K(1)$.

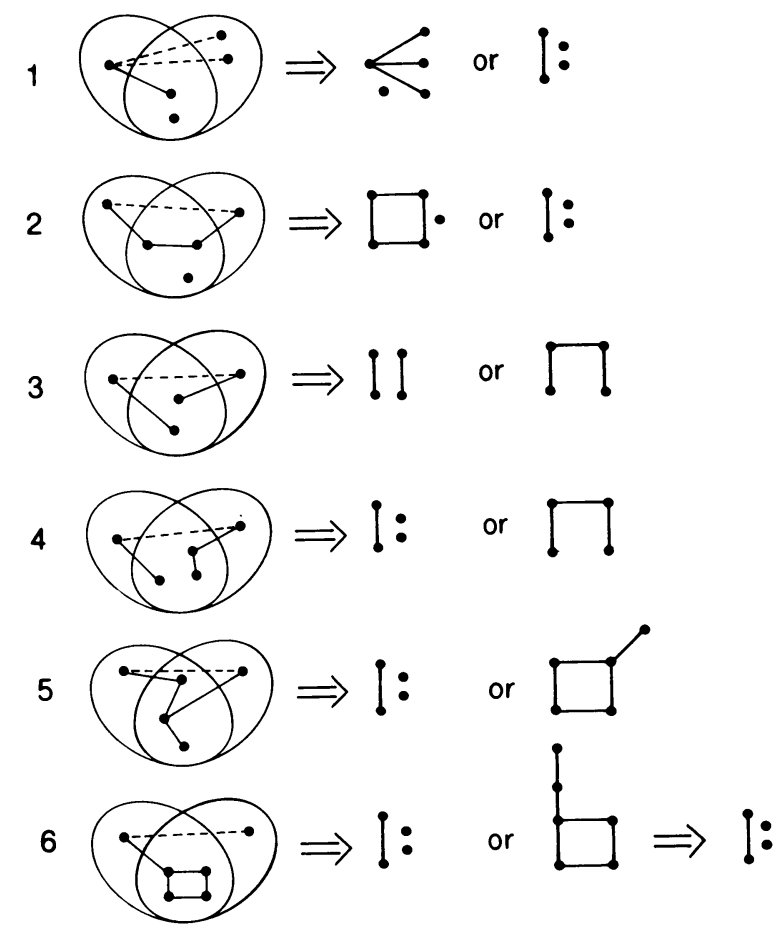

FIGURE 2

REMARK 2. We have written the proof of (i) in strict accordance with the definition of derivation. The reader will see that the following simplification in the presentation leads to no difficulty. If we wish to prove $\vdash_{m} G$ we may assume for contradiction that $\forall_{m} G$, and for a given amalgamation $Q$ we may suppose that one of $G_{1}, \ldots, G_{k}$ is derivable, where $G_{1}, \ldots, G_{k}$ are the possible outcomes of $\mathcal{Q}$ in which $G$ is not embeddable. If we can then derive $G$ this contradiction can be turned into a proof that $t_{m} G$. A formal justification of this can be given using the claim in the proof of Lemma D. From now on we will give only such simplified derivations. The reader should bear in mind that we are really dealing with derivable tuples of graphs.

(ii) See Figure 3. Assume that $\forall_{2} L(3)+K(1)$. Then from the amalgamation of $L(3)$ and $K(2)+K(1)$ over $K(2)$ we obtain $L(4)$. Then from the amalgamation of $L(4)$ and $K(2)+2 K(1)$ over $K(2)+K(1)$ we have $t_{2} L(3)+K(1)$. 
1

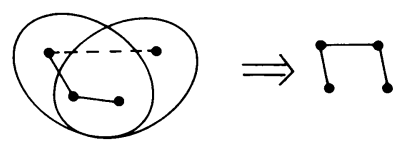

2

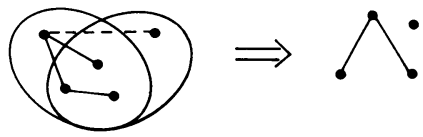

FIGURE 3

Now from (i) and (ii) we see that $\vdash_{2} L(3)+K(1), \vdash_{2} K(2)+K(1), \vdash_{2} K(2)+K(1)$ $+K(1)$ and $\vdash_{2} n K(1)+K(1)$ when Sublemma $2, \vdash_{2} G_{1} \vee \ldots \vee G_{n}$ implies $\vdash_{2}\left(G_{1}+K(1)\right) \vee \ldots \vee\left(G_{n}+K(1)\right)$. Thus from now on in deriving members of $\mathcal{G}(2)$ we may add copies of $K(1)$ whenever it is convenient.

(iii) See Figure 4. Assume that $\forall_{2} K(2)+K(2)$. From the amalgamation of $K(2)+2 K(1)$ and $L(3)+K(1)$ over $3 K(1)$ we get

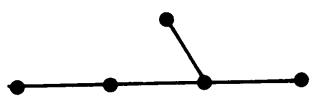

From the amalgamation of this graph with itself we get the result.

1

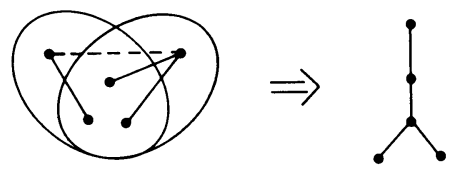

2

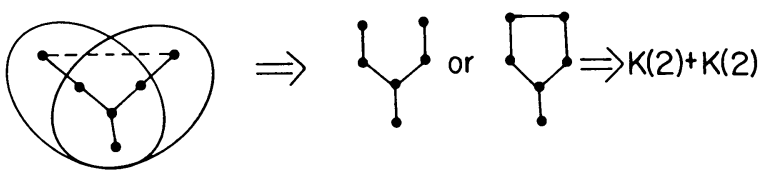

Figure 4

(iv) Assume that $\forall_{2} K(2)+L(3)$ for contradiction. See Figure 5 (1)-(6).

From consideration of these figures it is clear that $r_{2} K(2)+L(3)$. Now we see that $L(3)+K(2), K(2)+K(2), K(2)+K(1)+K(2)$ and $n K(1)+K(2) \in \mathcal{G}(2)$ so by Sublemma $2, \mathcal{G}(2)$ is closed under addition of $K(2)$, i.e. $\vdash_{2} G_{1} \vee \ldots \vee G_{n}$ implies $\vdash_{2}\left(G_{1}+K(2)\right) \vee \ldots \vee\left(G_{n}+K(2)\right)$.

(v) Assume for contradiction that $\forall_{2} L(3)+L(3)$. See Figure 6(1)-(5) and recall that $K(2)$ may be added to any derived graph.

From an examination of Figure 6 it is clear that $r_{2} L(3)+L(3)$.

This completes the proof of Lemma 1 when $m=2$.

5.2. For the remainder of this section we assume that $m>2$. Since $\vdash_{m} K\left(m^{\prime}\right)$ whenever $m^{\prime}<m$ it follows that $\mathcal{G}\left(m^{\prime}\right) \subset \mathcal{G}(m)$ whenever $m^{\prime}<m$, and by the induction hypothesis we have $\Sigma\left(m^{\prime}\right) \subset \mathcal{G}(m)$ when $m^{\prime}<m$. Thus $\vdash_{m} L(3)+L(3)$, and if $K(m)$ cannot be embedded in $H$ then $\vdash_{m} H$. 

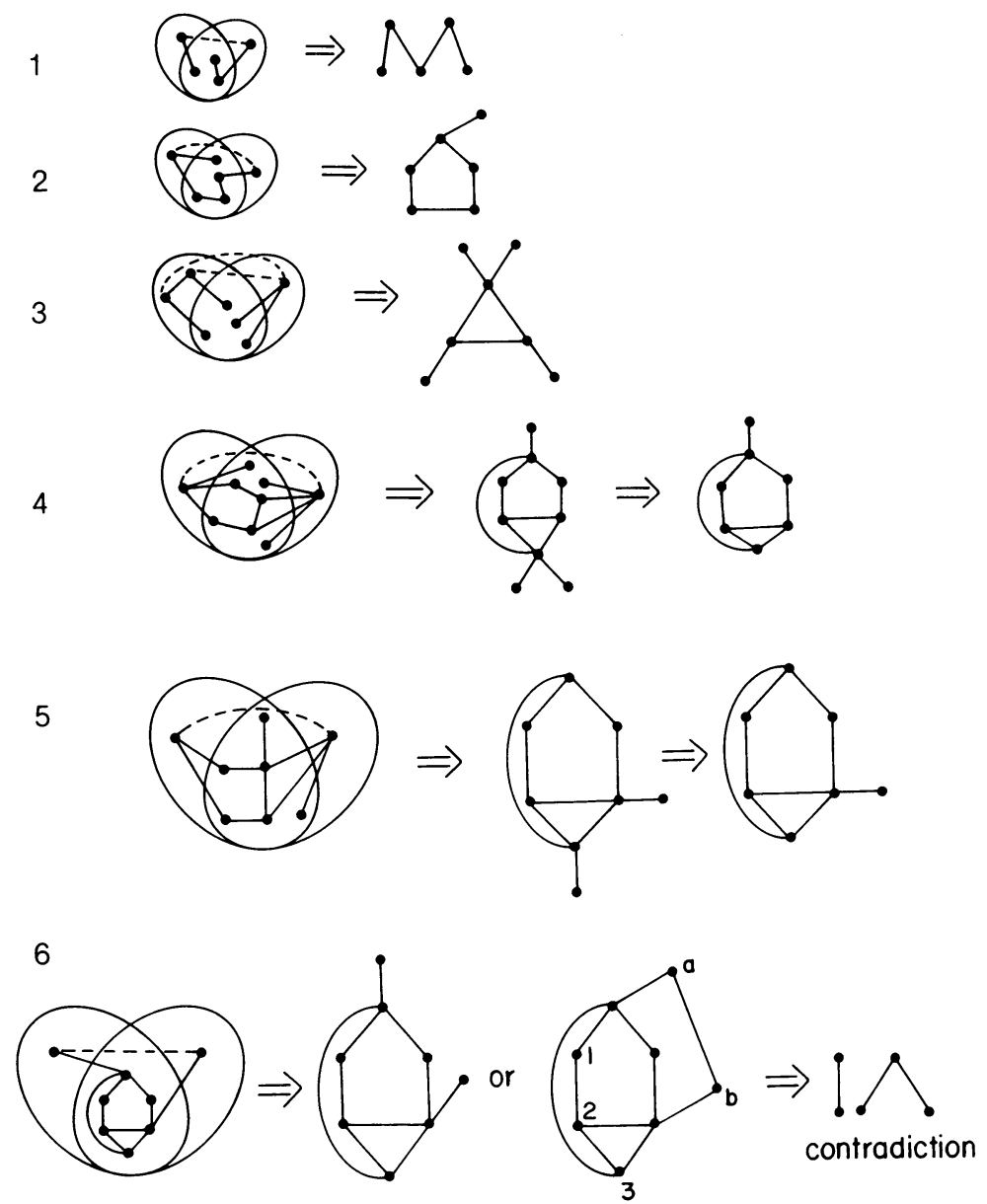

Figure 5

The proof has five parts:

(i) $\vdash_{m} K(m)+n K(1)$ for all $n$;

(ii) by induction on $k$ for $k \leqslant m-1$ we have $\vdash_{m} K(m)+n K(k)$ for all $n$;

(iii) $\vdash_{m} K(m)+L(3)$;

(iv) if $\vdash_{m} G_{1} \vee \ldots \vee G_{n}$ and $H \in \Sigma(m-1)$ then $\vdash_{m}\left(G_{1}+H\right) \vee \ldots \vee\left(G_{n}+\right.$ H);

(v) $\vdash_{m} K(m)+K(m)$.

We prove them in order.

(i) Assume for contradiction that $\forall_{m} K(m)+K(1)$. See Figure 7. From the amalgamation of $\{a\} \otimes K(m-1)$ and $\{b\}+K(m-1)$ over $K(m-1)$ we get $[(K(m), a),(K(2), a)]$ choosing $K(2)$ with $a$ as one vertex. Now in Figure 7(2) we see that if we amalgamate this graph and $[(K(m-1), a),(K(2), a)]+K(m-2)$, in which $K(m)$ cannot be embedded, over [ $K(m-1), a),(K(2), a)]$ we either have $K(m)+K(1)$ or the graph obtained from $[(K(m), a),(K(2), a)]$ by joining each vertex of a copy of $K(m-2)$ to a vertex $c$ of $K(m)$ different from $a$. Now by 

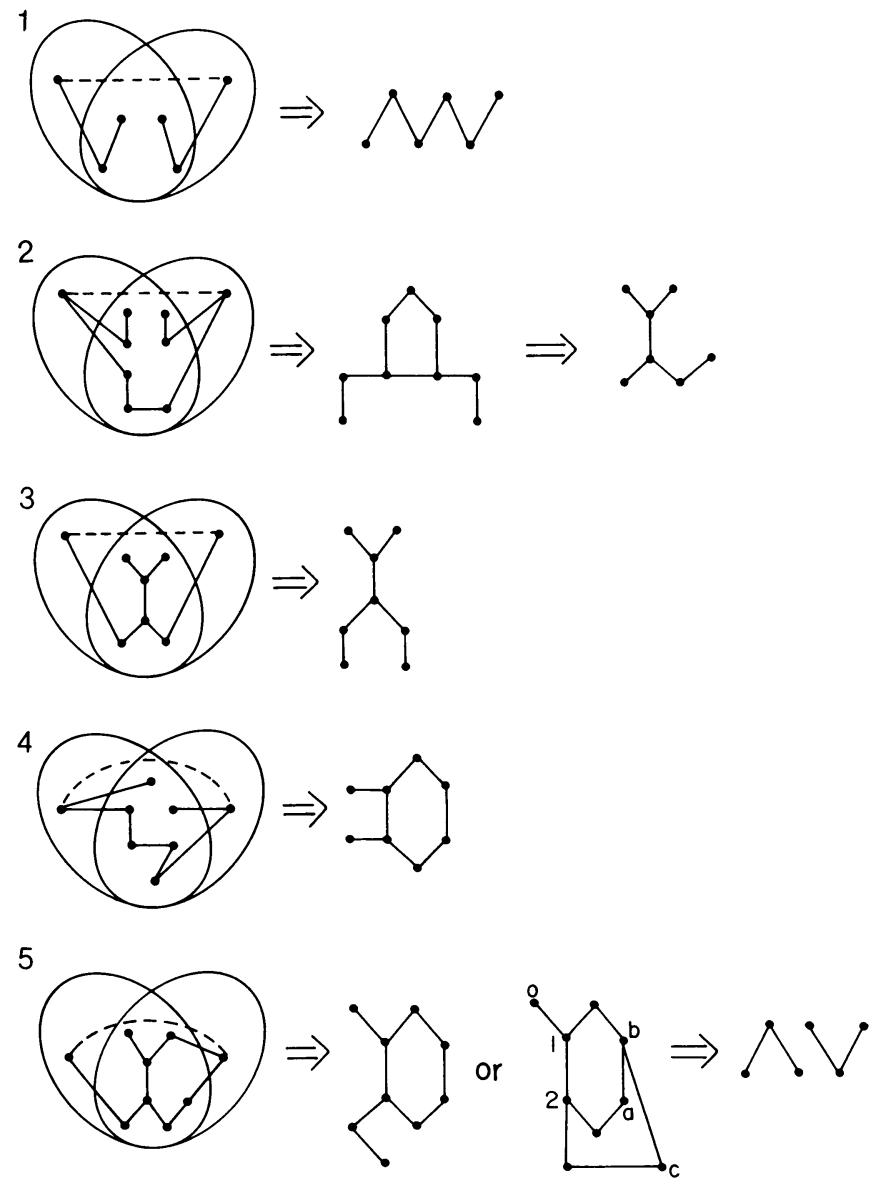

FIGURE 6

amalgamation of this graph and $K(m-1)+[(K(m-1), a),(K(2), a)]$ over $K(m-2)+[(K(m-1), a),(K(2), a)]$ we have a contradiction.

Therefore $\vdash_{m} K(m)+K(1)$. We may now use this argument, amalgamating over $X+(n-1) K(1)$, to prove by induction that $\vdash_{m} K(m)+n K(1)$ for all $n$.

This completes the proof of (i).

(ii) Using the same idea we now prove by induction on $k$ that for $1<k<m-$ $1, \vdash_{m} K(m)+n K(k)$. We have the result for $k=1$ from (i). Now assume that $k<m-1$ and that $\vdash_{m} K(m)+l K(k+1)+n K(k)$ for all $n$. We shall show that $\vdash_{m} K(m)+(l+1) K(k+1)+n K(k)$ for all $n$. Let $G$ be $l K(k+1)+n K(k)$, and assume for contradiction that $\forall_{m} G+K(m)+K(k+1)$. See Figure 8. From the amalgamation of $G+k K(k)+K(k)+K(m)$ and $G+k K(k)+K(m-1)+$ $K(k+1)$, the latter being in $\Sigma(m-1)$, over $G+k K(k)+K(m-1)+K(k)$ we have $\vdash_{m} H_{1}$. Now amalgamate $H_{1}$ and the graph $H_{2}$, formed by deleting one point of $K(m-1)$ in $H_{1}$ and adding $K(m-1)$, over the graph $L$. See Figure 8(3). Note 

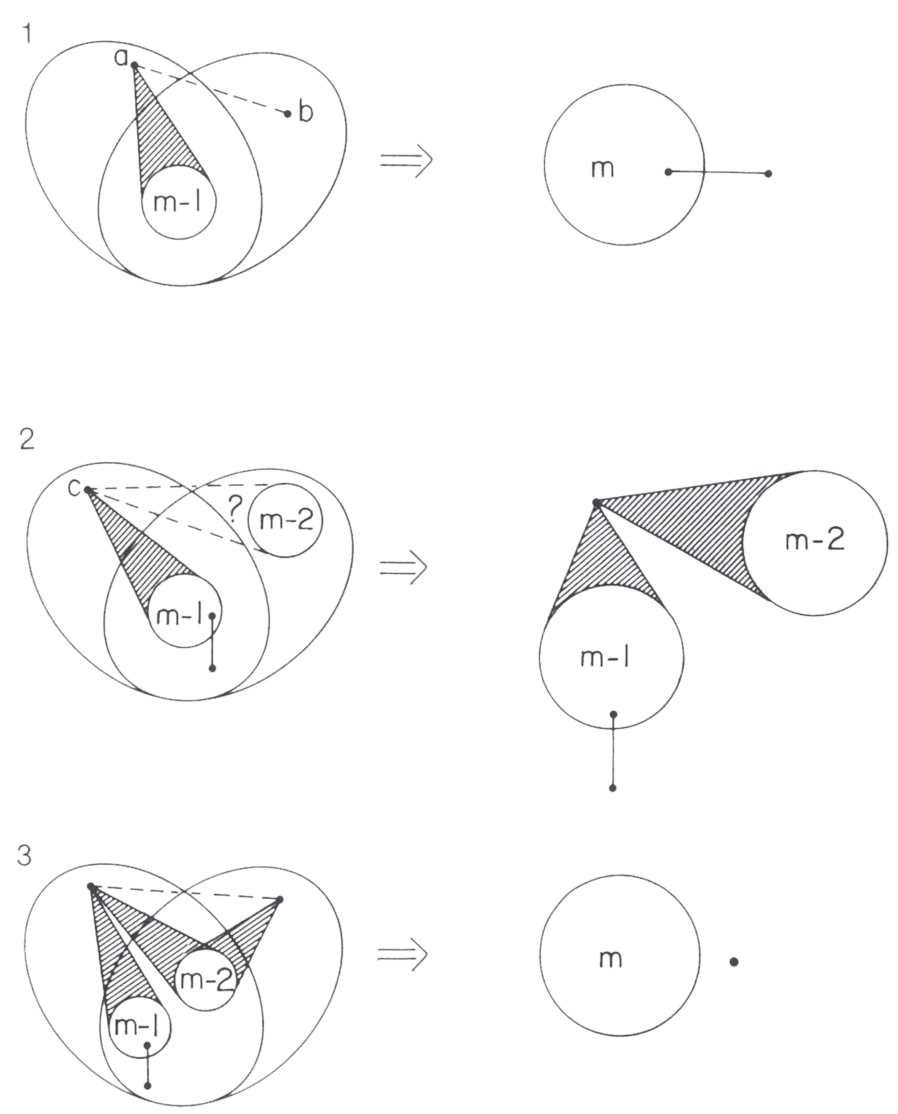

FIGURE 7

that $H_{2} \in \Sigma(m-1)$. If $a$ is joined to fewer than $m-(k+1)$ vertices of the copy of $K(m-1)$ then we are done for then we have $K(k+1)$ disjoint from $K(m)+G$. Therefore we have $\vdash_{m} H_{3}$. We now amalgamate $H_{3}$ and the graph shown in Figure $8(4)$ with $a$ deleted, over the common part. Note that the second graph is in $\Sigma(m-1)$. If $a$ is not joined to each vertex $d$ of $K(k)$ then $\{a\} \otimes K(m-1)$ and $\{d\} \otimes K(k)$ give the result. Otherwise $\{a\}, K(m-k-1)$ and $K(k)$ give $K(m)$ disjoint from the central copy of $K(k+1)$. Thus for $1 \leqslant k \leqslant m-1, \vdash_{m} K(m)+$ $n K(k)$ for all $n$.

(iii) $\vdash_{m} K(m)+L(3)$. For contradiction assume that $\forall_{m} K(m)+L(3)$. Recall that from (i) we already have $\vdash_{m} K(m)+n K(1)$ for all $n$. By amalgamation of $K(m)+$ $(n+2) K(1)$ and $L(3)+n K(1)+K(m-1)$, the latter being in $\Sigma(m-1)$, over $K(m-1)+(n+2) K(1)$ we have $\vdash_{m}[(K(m), a),(S(3), b)]+n K(1)$ for every $n$, where $b$ is a vertex at the tip of one of the rays of $S(3)$. See Figure $9(1)$. Now we follow the same line as in (i). The amalgamation shown in Figure 9(2) must have $a$ joined to each vertex of $K(m-2)$ since each vertex of $K(m-2)$ is connected to both $c_{0}$ and $c_{1}$. Note that deleting $a$ on the left of Figure 9(2) we obtain a graph in $\Sigma(m-1)$. Now we finish as in (i) by the amalgamation shown in Figure 9(3). 


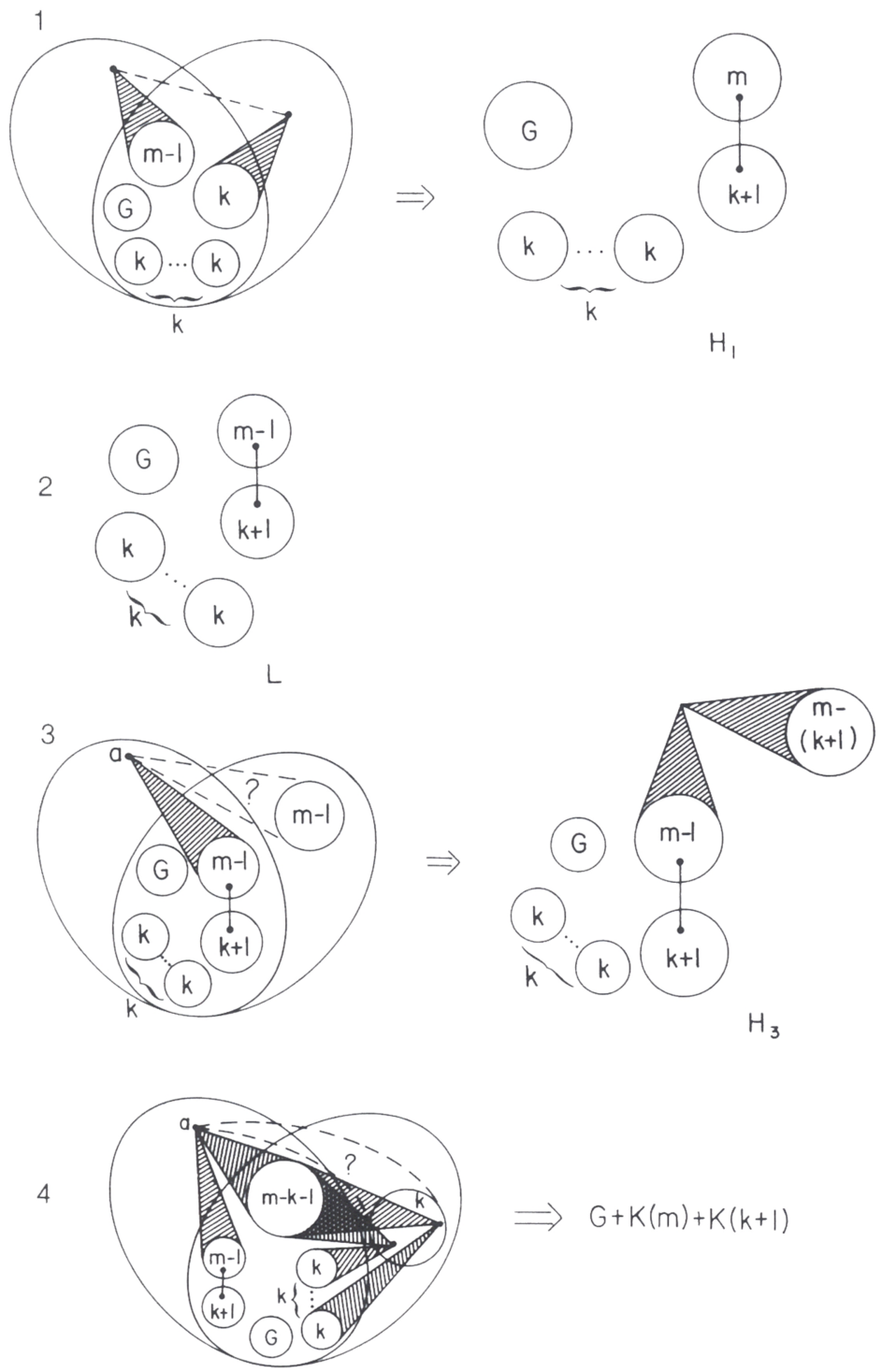

FIGURE 8

Notice that again deleting $a$ we have a graph in $\Sigma(m-1)$. This amalgamation gives $\vdash_{m} K(m)+L(3)$.

(iv) If $\vdash_{m} G$ and $H \in \Sigma(m-1)$ then $\vdash_{m} G+H$. Indeed if $\vdash_{m} G_{1} \vee \ldots \vee G_{n}$ and $H \in \Sigma(m-1)$ then $\vdash_{m}\left(G_{1}+H\right) \vee \ldots \vee\left(G_{n}+H\right)$.

The proof is like that of Lemma 2.

By (i), (ii), (iii) we have $r_{m} K(m)+K(m-1), K(m)+L(3), K(m)+n K(m-1)$ for all $n$, whence $\vdash_{m} K(m)+K(2)+K(1)$ and $\vdash_{m} K(m)+n K(1)$.

We now prove as in Lemma 2 by induction on derivation that if $r_{m-1} H_{1}$ $\vee \ldots \vee H_{n}$ then $\vdash_{m}\left(K(m)+H_{1}\right) \bigvee \ldots \bigvee\left(K(m)+H_{n}\right)$. Thus for $H \in \mathcal{G}(m-1)$ 

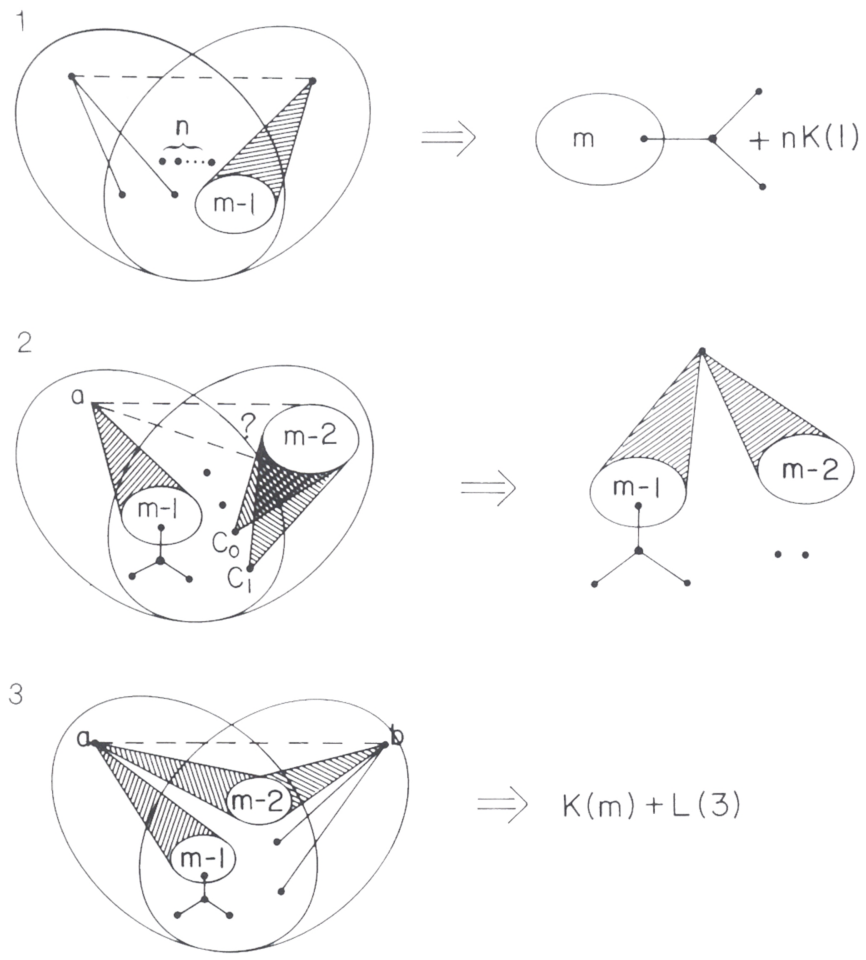

FigurE 9

we have $r_{m} K(m)+H$. Also by the induction hypothesis for $H \in \Sigma(m-1)$ we have

$$
\vdash_{m} L(3)+H, K(2)+K(1)+H, n K(1)+H
$$

since each of these graphs is in $\Sigma(m-1)$. Now fix $H \in \Sigma(m-1)$. Then $\vdash_{m-1} H$. We prove by induction on $q$ that if $\left\langle G_{1}, \ldots, G_{n}\right\rangle \in \mathcal{G}_{q}(m)$ there exists $p$ such that $\left\langle G_{1}+H, \ldots, G_{n}+H\right\rangle \in \mathcal{G}_{p}(m)$ as in Lemma 2. This completes the proof of (iv).

Finally we are able to complete the proof of Lemma 1 by:

(v) $\vdash_{m} K(m)+K(m)$. For contradiction assume that $\forall_{m} K(m)+K(m)$. From (ii) we have $\vdash_{m} K(m)+n K(m-1)$ for all $n \geqslant 1$. From the amalgamation of $K(m)+$ $(m-1) K(m-1)$ and a copy of itself over $m K(m-1)$ shown in Figure 10(1) we get $\vdash_{m} K(2) \otimes K(m-1)+(m-2) K(m-1)$. Now from the amalgamation in Figure $10(2)$ of $K(2) \otimes K(m-1)+(m-2) K(m-1)$ and $K(m)+(m-1) K(m-1)$ over $m K(m-1)$ we obtain $\vdash_{m} K(3) \otimes K(m-1)+(m-3) K(m-1)$ and by repeating this argument we see that $\vdash_{m} K(m) \otimes K(m-1)$ unless $\vdash_{m} K(m)+K(m)$. Now by (iv) we see that

$$
\vdash_{m}[(K(m), a),(K(m-1), a)]+K(2) \otimes K(M-2)
$$

and

$$
\begin{aligned}
& \vdash_{m}\left[\left(K(m-1), a_{1}\right)\left(K(m-1), a_{1}\right)\right] \\
& +\left[\left(\left[\left(K(m), a_{2}\right),\left(K(2), a_{2}\right)\right], a_{3}\right),\left(K(m-1), a_{3}\right)\right]
\end{aligned}
$$



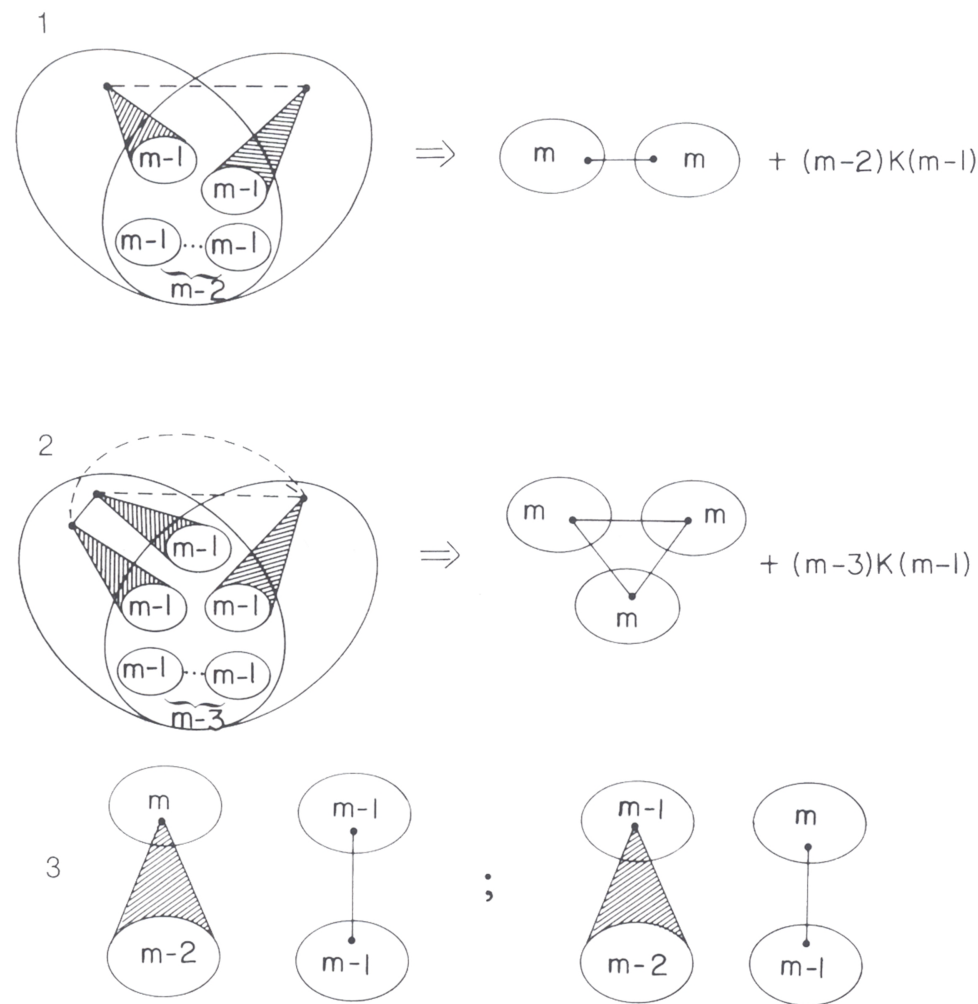

4

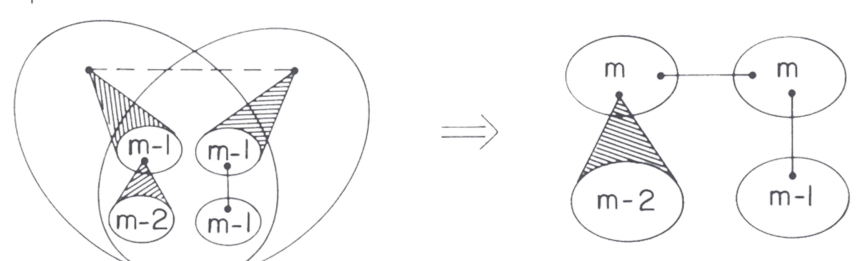

$\mathrm{H}$

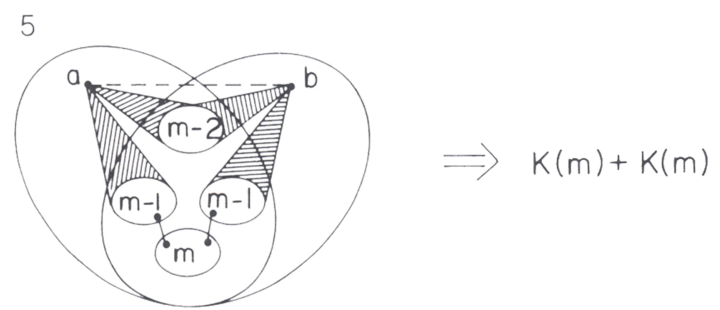

Figure 10 
where $K(2)$ is $\left\{a_{2}, a_{3}\right\}$. See Figure $10(3)$. From the amalgamation shown in Figure 10(4) we obtain the graph $H$. Finally the amalgamation shown in Figure 10(5) gives $r_{m} K(m)+K(m)$. Notice that in Figure 10(5) the result of deleting either $a$ or $b$ gives $H$. If $a$ is not joined to $b$ we are done immediately. Otherwise $K(m-2) \cup$ $\{a\} \cup\{b\}$ is $K(m)$ disjoint from $K(m)$ in the common part.

This completes the proof of Lemma 1.

6. Proof of Lemma 3. In this section we prove the following:

LEMMA 3. $\vdash_{m} K(m) \otimes K(m-1)$ and if $m>3, r_{m} K(m) \otimes L(3)$. Also $\vdash_{m} L(3) \otimes$ $K(m-1)$ and if $m>3$ then $\vdash_{m} L(3) \otimes L(3)$. In this section we consider two separate cases: $m=2$ and $m>2$.

We thank Ron Morrow for his collaboration in the proofs that $L(3) \otimes K(m-1)$ and $L(3) \otimes L(3)$ are in $\mathcal{G}(3)$. His proof that $\vdash_{3} K(2) \otimes K(2)$ provided a key idea for these proofs.

6.1. Let $m=2$. We must show that $L(4) \cong K(2) \otimes K(1)$ and $L(3) \otimes K(1)$ are in $\mathcal{G}(2)$. The amalgamation showing that $t_{2} L(4)$ is shown in Figure 11(1). We amalgamate two copies of $L(3)+K(1)$ over $3 K(1)$. By repeated use of this idea we have $r_{2} L(n)$ for all $n \geqslant 3$. The amalgamation of two copies of $L(n)$ over $2 L(n-2)$ $+K(1)$ shown in Figure 11(2) gives $L(2 n-2)$.
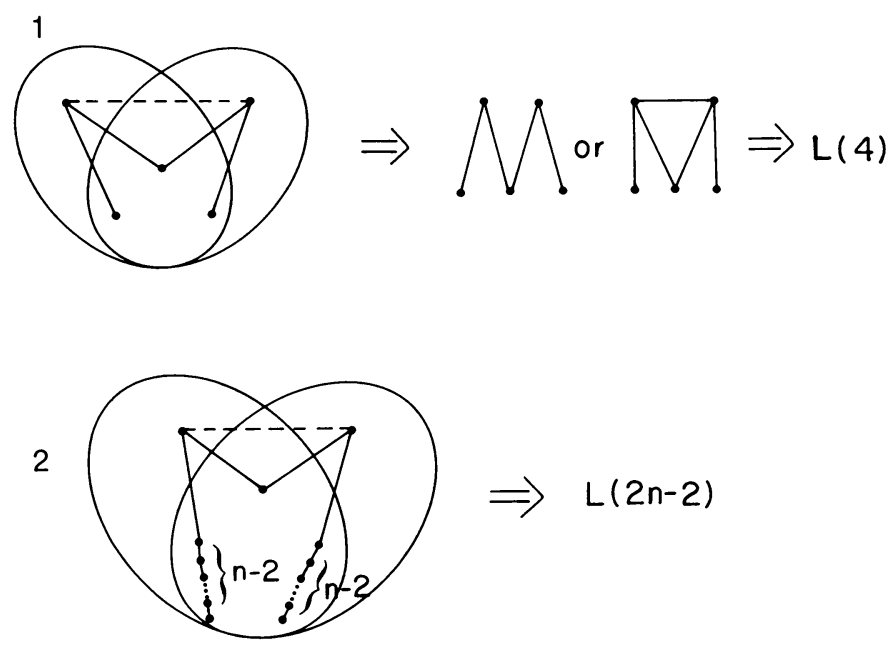

FIgURE 11

Next we prove $\vdash_{2} S(3)$.

Following the method of presentation of Lemma 1 we assume for contradiction that $\forall_{2} S(3)$. From the amalgamation shown in Figure 12(1) of two copies of $L(5)$ over $2 K(2)$ we obtain the graph $G$. Then the amalgamation of $G$ and $L(3)+2 K(1)$ over $K(2)+2 K(1)$ gives $S(3)$. See Figure 12(2). 

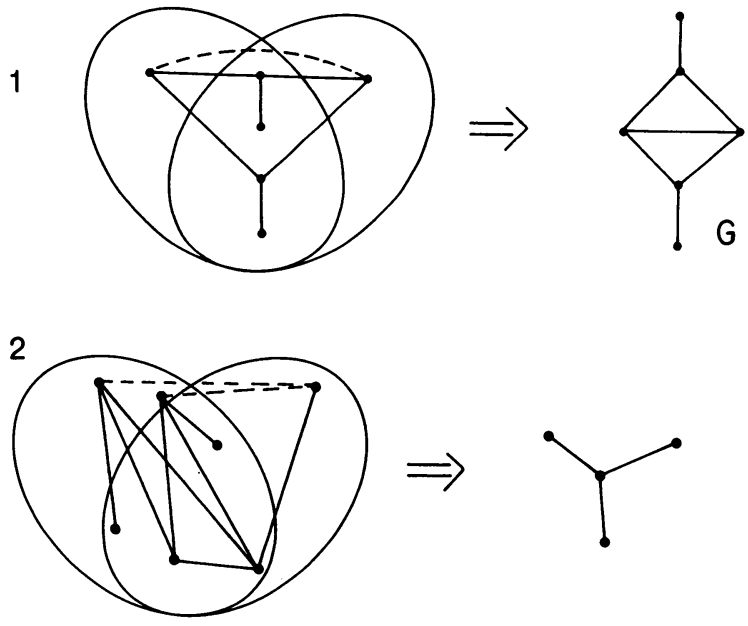

Figure 12

Now assume for contradiction that $\forall_{2} L(3) \otimes K(1)$. Amalgamating $S(3)+L(4)$ and $L(8)$ over $L(3)+L(4)$ as in Figure 13(1) we obtain G. Finally in Figure 13(2) we obtain $L(3) \otimes K(1)$ by the amalgamation of $G+K(1)$ and $L(5)+L(3)$ over $2 L(3)+K(1)$.

Thus $\vdash_{2} L(3) \otimes K(1)$.
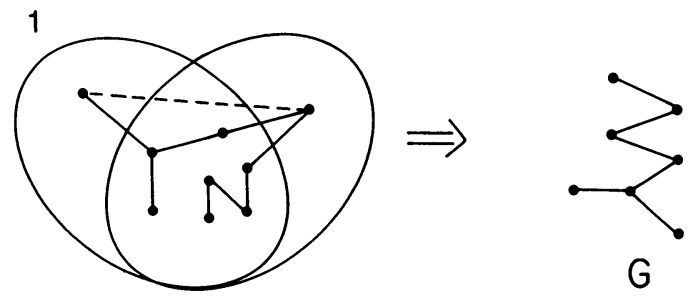

2

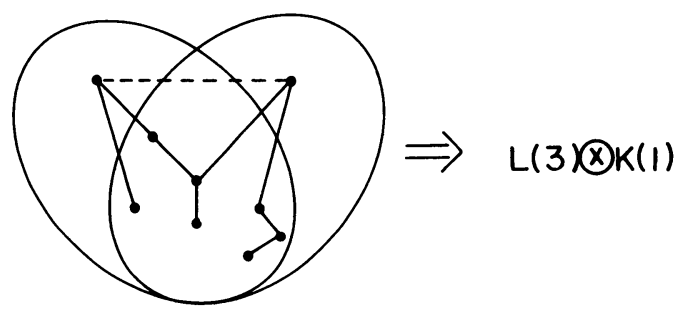

Figure 13 
This completes the proof of Lemma 3 if $m=2$.

6.2. Assume that $m \geqslant 3$. We must prove that $K(m) \otimes K(m-1), L(3) \otimes$ $K(m-1), L(3) \otimes L(3)$ and $K(m) \otimes L(3)$ are all in $\mathcal{G}(m)$. The proof is broken into ten parts. In some of these we must distinguish between the cases $m=3$ and $m>3$.

(i) $\vdash_{m} K(m) \otimes K(1)$.

(ii) If $\vdash_{m} K(m) \otimes K(k)$ and $1 \leqslant k \leqslant m-2$ then $\vdash_{m} G_{1} \vee \ldots \vee G_{n} \Rightarrow$ $\vdash_{m}\left(G_{1} \otimes K(k)\right) \vee \ldots \vee\left(G_{n} \otimes K(k)\right)$.

(iii) For $1 \leqslant k \leqslant m-2, K(m) \otimes K(k) \in \mathcal{G}(m)$. This will be proved by induction on $k$.

(iv) $\vdash_{m} K(1) \otimes L(3)$.

(v) If $m>3$ then $\vdash_{m} K(m) \otimes L(3)$. Here the proof follows the same line as in (iii).

(vi) $\vdash_{m} K(m) \otimes K(m-1)$. This part breaks down into a number of subparts.

(1) $\vdash_{m}[(K(m), a),(K(m), a)]$.

(2) For any graph $G$ and $a_{0} \in|G|$ such that $\vdash_{m}\left[\left(G, a_{0}\right),\left(K(m), a_{0}\right)\right]$ we have

$$
\vdash_{m}\left[\left(\left[\left(G, a_{0}\right),\left(K(m), a_{0}\right)\right], a_{1}\right),\left(K(m), a_{1}\right)\right]
$$

where $a_{0}, a_{1}$ are distinct vertices of $K(m)$.

(3) For any graph $G$, and $a \in|G|$ if $\vdash_{m}[(G, a),(K(m), a)]$ then

$$
\vdash_{m}[([(G, a),(K(m), a)], a),(K(m), a)] .
$$

(4) $\vdash_{m} K(l) \otimes K(m-1)$ for $1 \leqslant l \leqslant m$ by induction on $l$.

Refer to Figure 14(1)-(3) for illustrations of subparts (1), (2), (3).

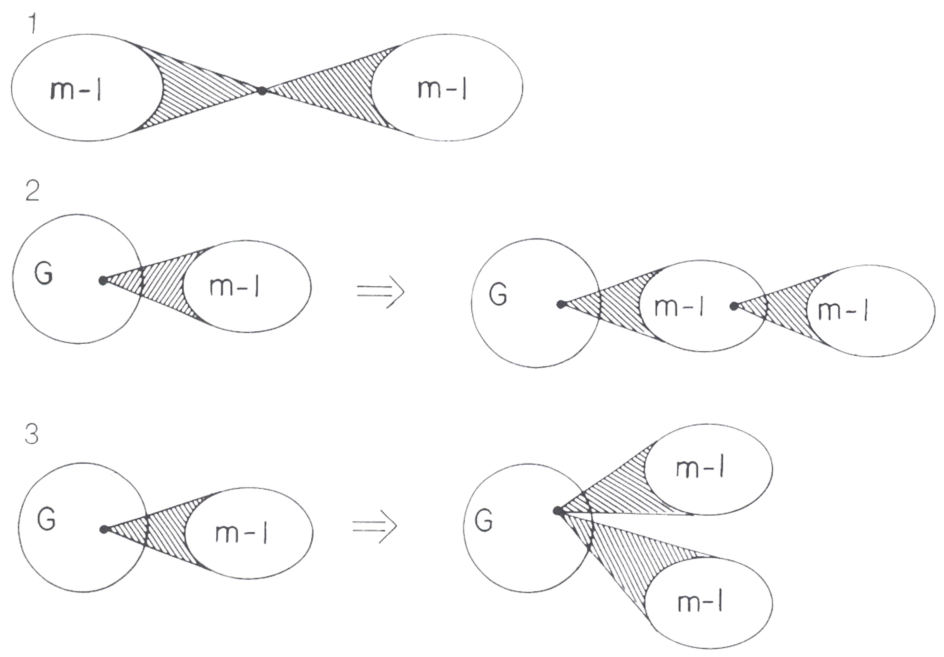

FIGURE 14

(vii) $\vdash_{m} L(3) \otimes K(m-1)$. This part has two subparts: (1) $m=3$ and (2) $m>3$. (viii) If $\vdash_{3} G_{1} \vee \ldots \vee G_{n}$ then $\vdash_{3}\left(G_{1} \otimes K(2)\right) \vee \ldots \vee\left(G_{n} \otimes K(2)\right)$.

(ix) $\vdash_{3} K(3) \otimes L(3)$. Here we apply the same technique as in (vi). First we prove $(1) \vdash_{3}[(\{a\} \otimes L(3), a),(\{a\} \otimes L(3), a)]$. 
(2) If $\vdash_{3}[(G, a),(\{a\} \otimes L(3), a)]$ then

$$
r_{3}[([(G, a),(\{a\} \otimes L(3), a)], b),(\{b\} \otimes L(3), b)] .
$$

(3) If $\vdash_{3}[(G, a),(\{a\} \otimes L(3), a)]$ then

$$
r_{3}[([(G, a),(\{a\} \otimes L(3), a)], a),(\{a\} \otimes L(3), a)] .
$$

Here $\{a\}$ denotes the one element graph, and $b$ is the unique vertex of $\{a\} \otimes L(3)$ which is the image of $a$ under an automorphism moving $a$.

(4) For $k=1,2,3, r_{3} K(k) \otimes L(3)$.

See Figure 15 for illustrations of subparts (1)-(3).

1

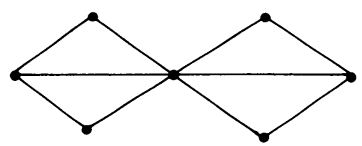

2

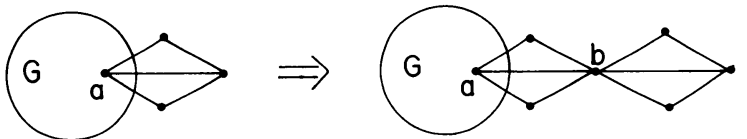

3

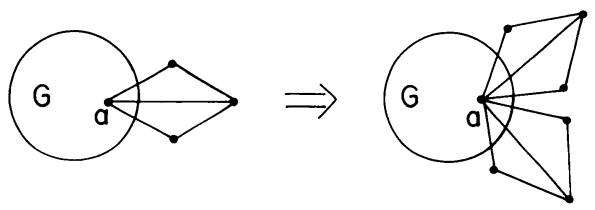

FIGURE 15

Finally we complete the proof of Lemma 3 with

$(\mathrm{x}) \vdash_{3} L(3) \otimes L(3)$.

(i) First we show that $\vdash_{m}[(K(m), a),(K(2), a)]$. As shown in Figure 16(1) we amalgamate $K(m)+K(1)$ and $[(K(m-1), a),([(K(m-1), b),(K(2), b)], a)]$, the latter belonging to $\Sigma(m-1)$ over $K(m-1)+K(1)$. Here $a, b$ are distinct vertices of $K(m)$. If $c$ is not joined to a vertex $d$ in the copy of $K(m-2)$ we are done with the left-hand copy of $K(m-1)$ together with the edge $a, d$. Otherwise $K(m-2)$, $a, c$ and $f$ give $\vdash_{m}[(K(m), a),(K(2), a)]$. Now we repeat the argument as shown in Figure 16(2) to attach a copy of $K(1)$ to $m-1$ vertices of $K(m)$ giving the graph $G$. Next we amalgamate $G$ and $K(m-1) \otimes K(1)+K(1)$ as in Figure 16(3) to get either the desired result or the graph $H$. Finally the amalgamation of Figure 16(4) gives $K(m) \otimes K(1)$.

(ii) Now $\vdash_{m} L(3) \otimes K(k)$ since $L(3) \otimes K(k) \in \Sigma(m-1)$ when $k<m-1$. If $\vdash_{m} K(m) \otimes K(k)$ then we may apply the reasoning of Lemma 4 and obtain:

$$
\vdash_{m} G_{1} \vee \ldots \vee G_{n} \Rightarrow r_{m}\left(G_{1} \otimes K(k)\right) \vee \ldots \vee\left(G_{n} \otimes K(k)\right)
$$

Once we have $r_{m} K(m) \otimes K(k)$ for $k<m-1$ this allows us to attach a copy of $K(k)$ to any vertex of any graph we use in an argument. 

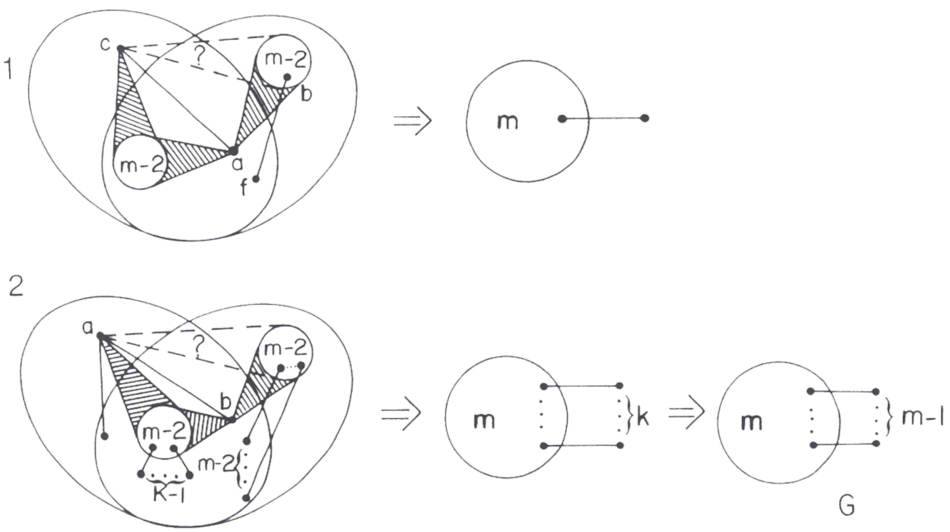

3
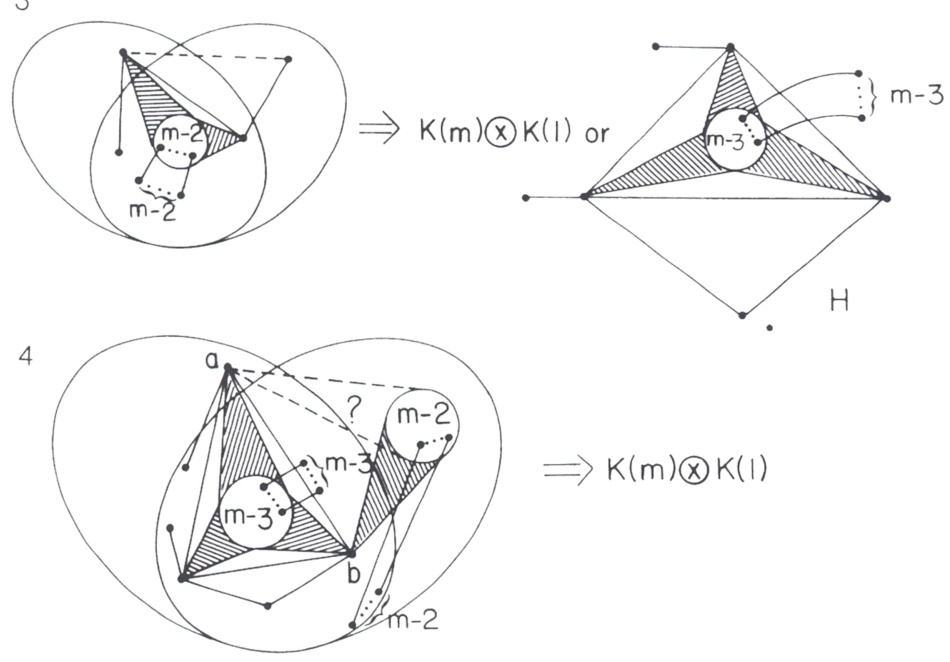

FIGURE 16

(iii) Now we prove by induction on $k$ that if $1 \leqslant k \leqslant m-2$ then $\vdash_{m} K(m) \otimes$ $K(k)$. Clearly from (i) we have the case $k=1$. Further from the induction hypothesis on $k$ and (ii), if $\vdash_{m} G_{1} \vee \ldots \vee G_{n}$ then $\vdash_{m}\left(G_{1} \otimes K(k-1)\right) \vee \ldots \vee\left(G_{n}\right.$ $\otimes K(k-1)$ ). An obvious consequence is that if $G$ has been derived and $a \in|G|$ then we may assume that $[(G, a),(\{a\} \otimes K(k-1), a)]$ is also derivable.

We have to show $\vdash_{m} K(m) \otimes K(k)$. We first argue that $\vdash_{m}[(K(m), a)$, $(K(k+1), a)]$. To see this choose the largest $l \leqslant k-1$ such that the graph $G_{l}$ shown in Figure 17(1) satisfies $\vdash_{m}[(K(m), a),(K(k+1), a)] \vee G_{l}$. When $l=0, G_{l}$ is $K(m)$ so $l$ is well defined. The amalgamation shown in Figure 17(2) gives $l=k-$ 1. One of the two graphs being amalgamated is obtained by attaching $K(k-1)$ to the vertex $c$ of $G_{l}$ and adding $K(k)$. The other is a member of $\Sigma(m-1)$ since $l \leqslant k-1$ and $(m-k-1)+(k-1)+1=m-1$ and $k \leqslant m-2$. If $a$ is not joined to every vertex of $K(m-k-1)$ then at once we get $[(K(m), a)$, $(K(k+1), a)]$ because if $b$ is the vertex of $K(m-k-1)$ in question we have the 


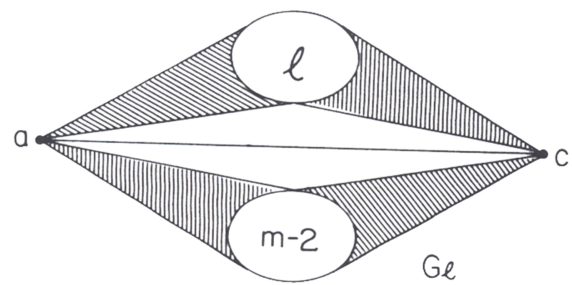

2

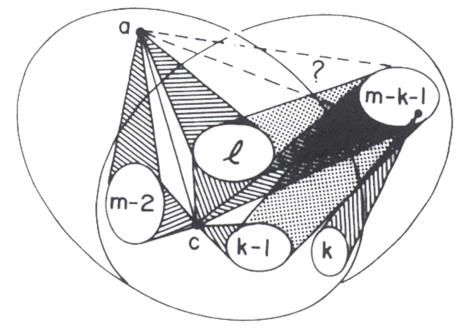

3

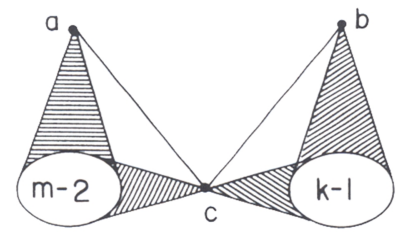

4

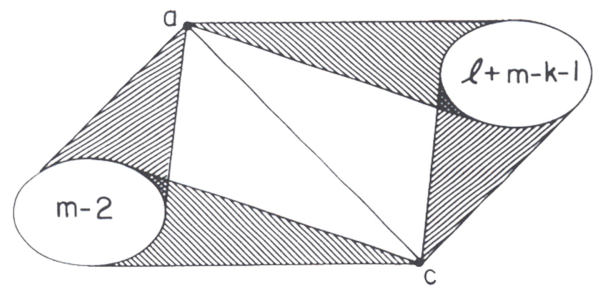

5

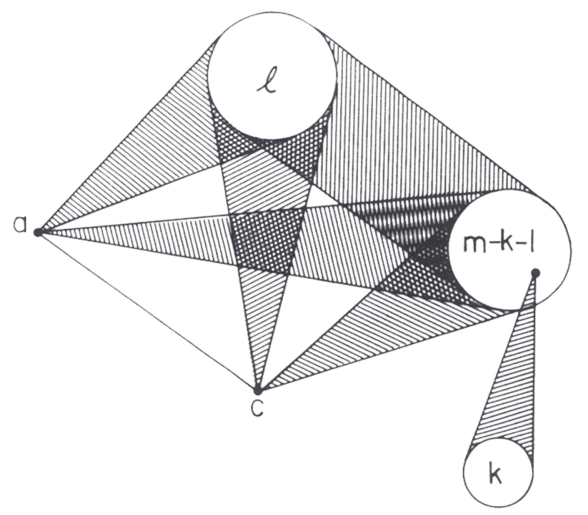

Figure 17 
graph of Figure 17(3). The other possibility is that $a$ is joined to every vertex of $K(m-k-1)$. It is then clear that in fact $l=k-1$ since then we have the subgraph $G_{l+m-k-1}$ in Figure 17(4). Now the graph shown in Figure 17(5) is just $[(K(m), a),(K(k+1), a)]$ and so the amalgamation of Figure 17(2) in fact gives $\vdash_{m}[(K(m), a),(K(k+1), a)]$.

Now we attach copies of $K(k)$ to $m-1$ vertices of $K(m)$ one at a time. The slight change in the iteration is that we start by joining a copy of $K(k)$ to each vertex of $K(l)$ and $K(m-k-1)$, to $a$ and to the appropriate number $n \leqslant m-2$ of vertices of $K(m-2)$. That is we choose $l$ to be the largest number such that the graph $G_{l}$ of Figure 18(1) is such that $\vdash_{m} H \vee G_{l}$ where $H$ is the graph with $K(k)$ suspended from $n+2$ vertices of $K(m)$, as in Figure 18(1). Then the amalgamation of Figure 18(2) gives us as above that $l=k-1$ and thus that $\vdash_{m} H$. See Figure $18(3)-(5)$
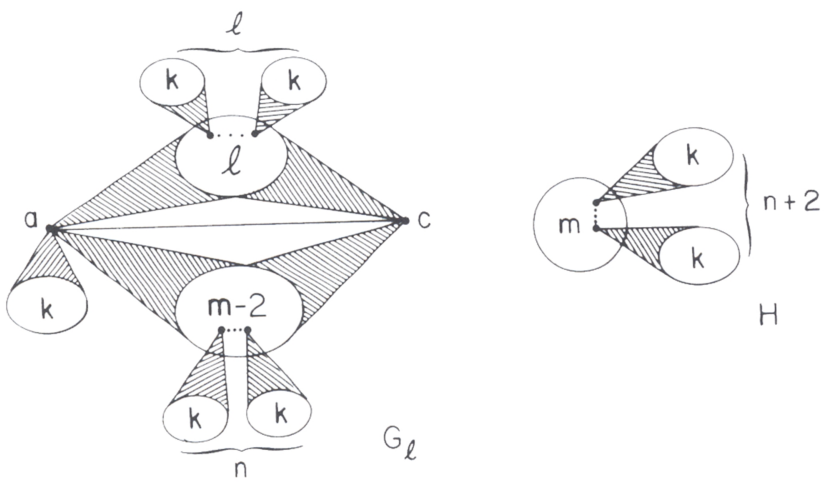

$H$

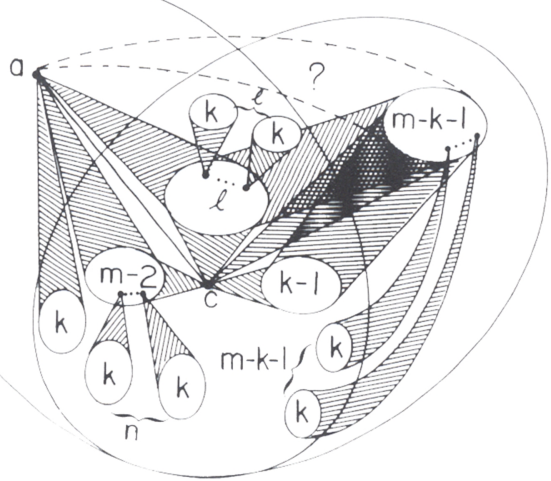


3

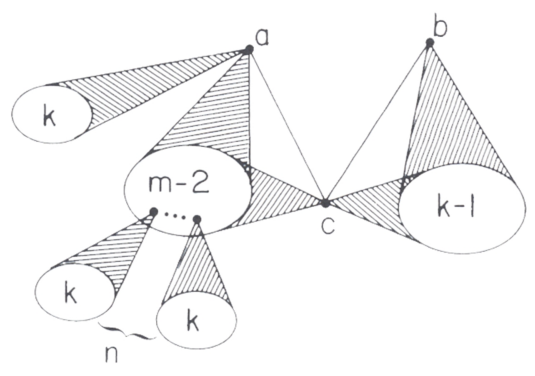

4

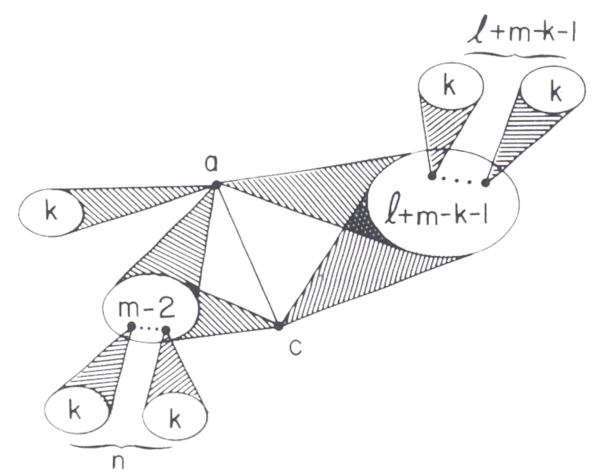

5

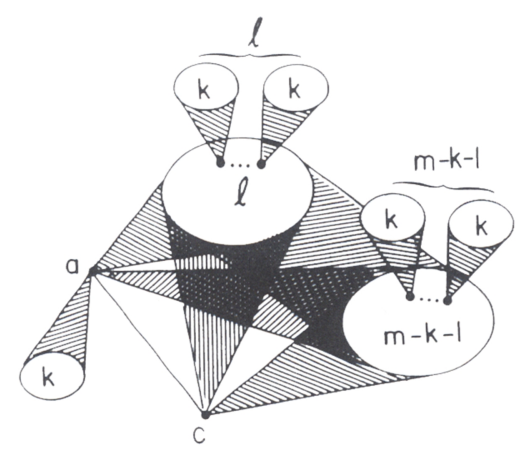

FigURE 18

Finally we perform the amalgamations of Figure 19 to obtain $K(m) \otimes K(k)$. We first amalgamate two copies of the graph with $K(k)$ attached to $m-1$ vertices of $K(m)$ as in Figure 19(1). This gives either $K(m) \otimes K(k)$ or this graph with one edge between two of the copies of $K(k)$. Then we repeat the argument above letting $l \leqslant k-1$ be largest such that $\vdash_{m}(K(m) \otimes K(k)) \vee G_{l}$ where $G_{l}$ is the graph of Figure 19(2). The amalgamation of Figure 19(3) then gives the result. If $a$ is not joined to some vertex $b$ of $K(m-k-1)$ we have $K(m) \otimes K(k)$ as in Figure 19(4). Otherwise we see that $l=k-1$ by Figure 19(5) and that $K(m) \otimes K(k)$ then can be embedded, Figure 19(6). This completes the proof that $\vdash_{m} K(m) \otimes K(k)$ and (iii) follows. 

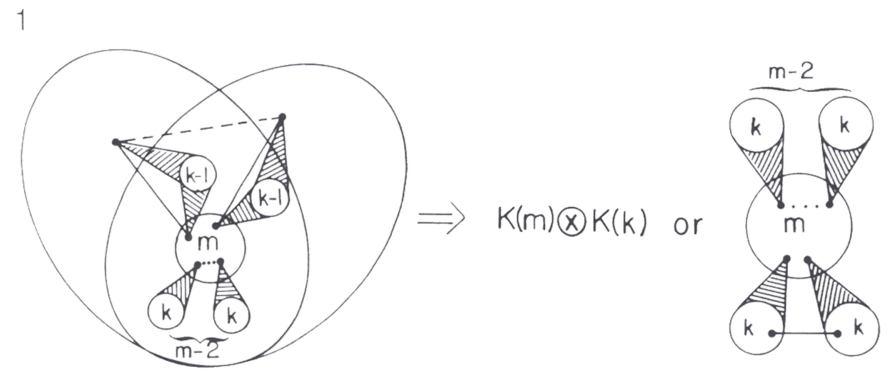

2

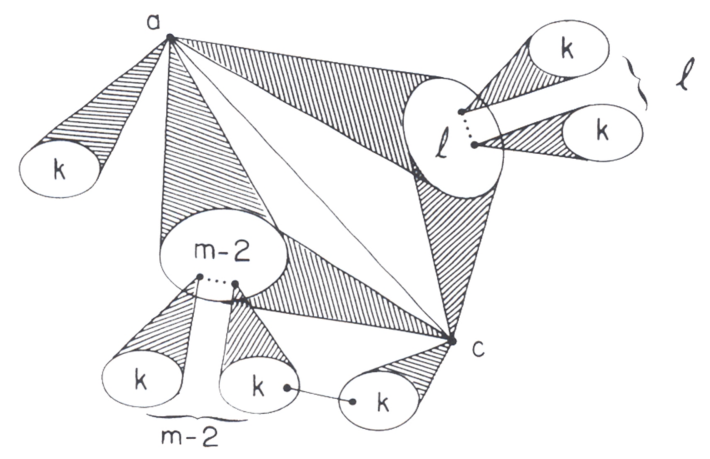

3

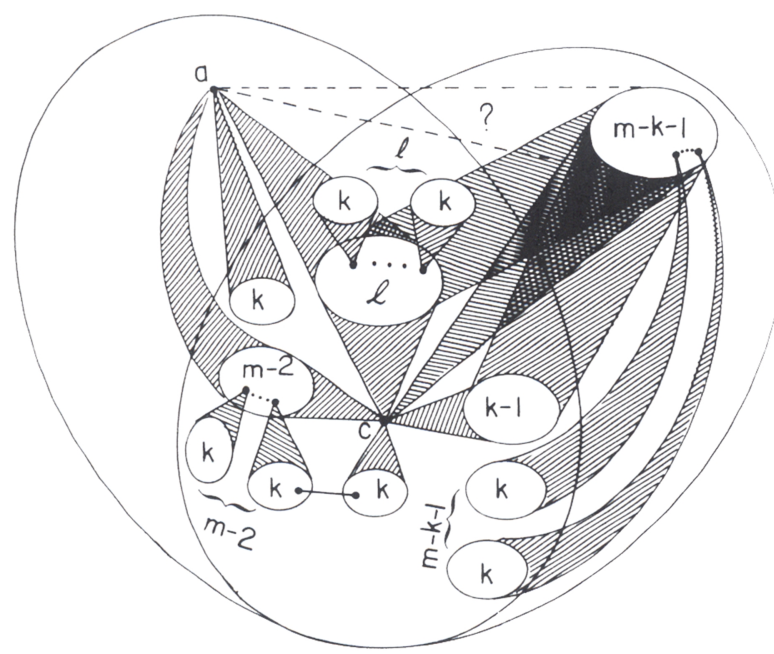




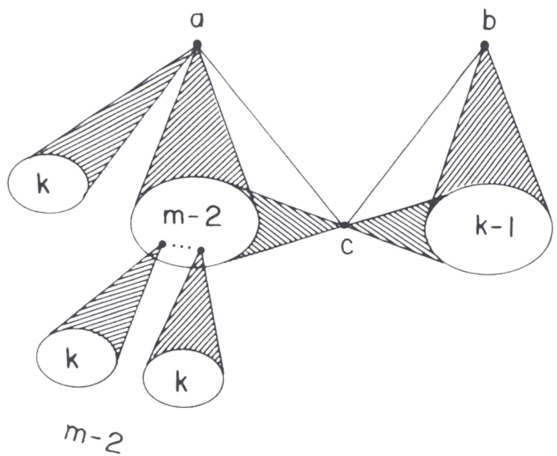

5

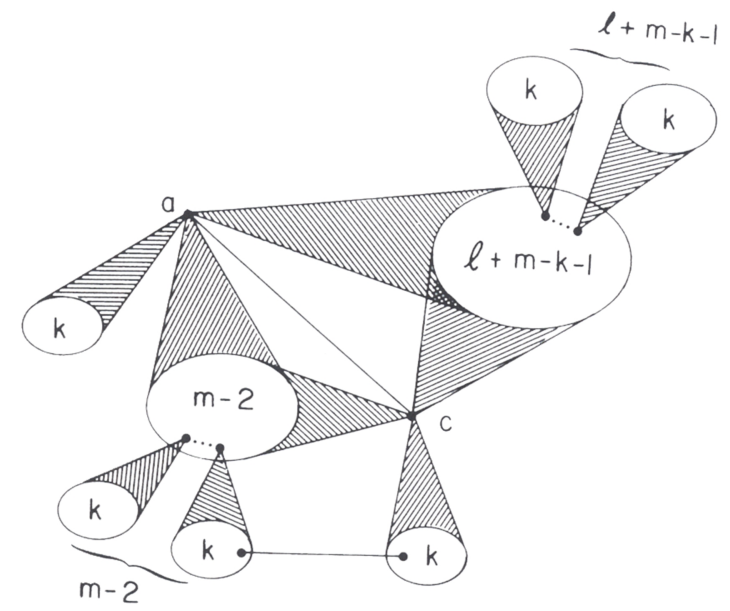

6

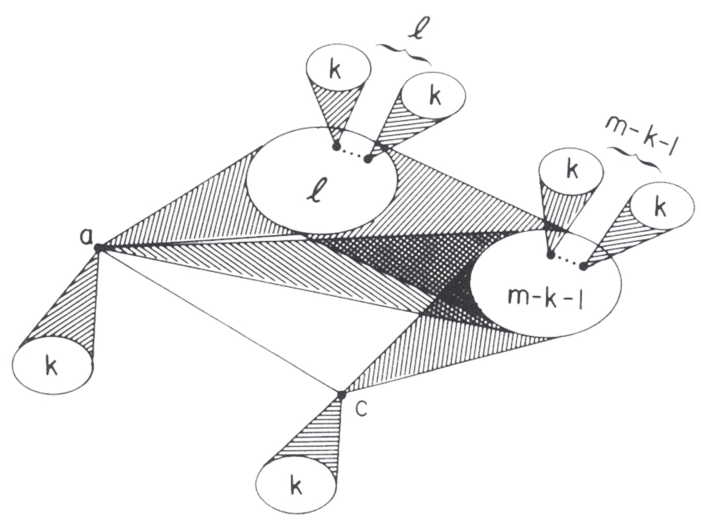

Figure 19 
(iv) $f_{3} K(1) \otimes L(3)$. This is immediate from the amalgamation of $[(K(3), a)$, $(K(2), a)]+K(1)$ and $[([(K(3), b),(K(2), b)], b),(K(2), b)]$ over $K(2)+2 K(1)$. The graphs which we amalgamate belong to $\mathcal{G}(3)$ since by (i) and (ii) if $\vdash_{3} G$ and $a \in|G|$ then $\vdash_{3}[(G, a),(K(2), a)]$. The amalgamation is shown in Figure 20.

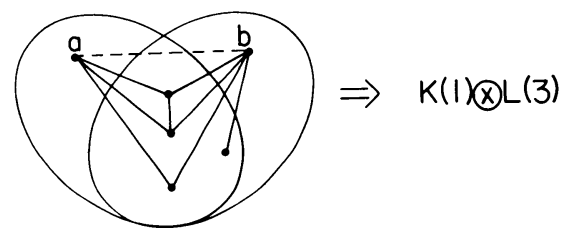

FIGURE 20

(v) $\vdash_{m} K(m) \otimes L(3)$ for all $m>3$.

The proof follows very closely that given in detail for (iii). Note that from (iii), $\vdash_{m} K(m) \otimes K(2)$, and from (ii), $K(2)$ may be attached to any vertex of any graph. The figure corresponding to Figure 17 is Figure 21 . Here $l$ is 0 or 1 and is defined to be the largest number $\leqslant 1$ such that $\vdash_{m} G_{l} \vee H$ where $H$ is obtained by attaching a copy of $L(3)$ to a vertex of $K(m)$. The argument of (iii) is then easily modified to handle this part, with $L(3)$ playing the role of $K(k)$. Corresponding changes to Figure 18 and Figure 19 and the associated arguments yield that $\vdash_{m} K(m) \otimes L(3)$. We leave the details to the reader.

(vi) We now set about proving $\vdash_{m} K(m) \otimes K(m-1)$.

By (iii) we have $\vdash_{m} K(m) \otimes K(m-2)$ and so by (i), in an argument we may attach $K(m-2)$ to any vertex of any graph in a derivation.

(1) We first prove that the graph $[(K(m), a),(K(m), a)]$ belongs to $\mathcal{G}(m)$. From the amalgamation of two copies of $[(K(m), a),(K(m-1), a)]$ over $[(K(m-1), a)$, $(K(m-1), a)$ ] shown in Figure 22(1) we obtain the graph we want or the graph $G$. Now the amalgamation of Figure 22(2) completes the argument. Notice that when $a$ is deleted we have a graph obtained by attaching successive copies of $K(k)$ for $k=m-2$ or 1 to derived graphs. Similarly with $b$ deleted we have $G$ with copies of $K(m-2)$ attached to two of its vertices.

(2) Now suppose that $G$ is any graph and $a \in|G|$ is such that $\vdash_{m}[(G, a),(K(m), a)]$. We want to show that $\vdash_{m}[([(G, a),(K(m), a)], b),(K(m), b)]$ where $a, b$ are distinct. This fact is clear from (1) and the amalgamation shown in Figure 23. When $c$ is deleted we obtain the graph by attaching copies of $K(m-2)$ to $a$ and $b$. Let $G^{-}$result from $G$ by deleting $a$. When $a$ is deleted from Figure 23 we have the union of $G^{-}$and a graph obtained from $[(K(m), a),(K(m), a)]$ by attaching copies of $K(m-2)$ appropriately. If the edge $a, c$ is not in the amalgamation then $b, K(m-2)$ and $c$ give the attached $K(m-1)$. Otherwise $a, c$, $K(m-2)$ give $K(m-1)$ attached at $a$, and $K(m-1)$ is attached to $c$. 


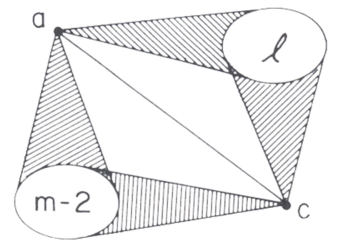

2

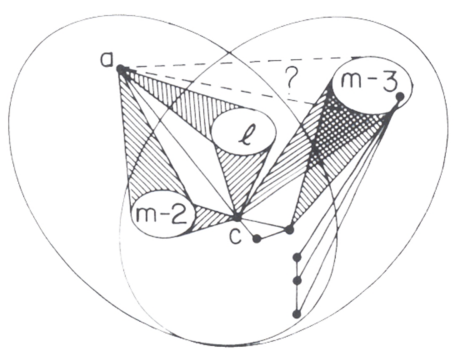

3

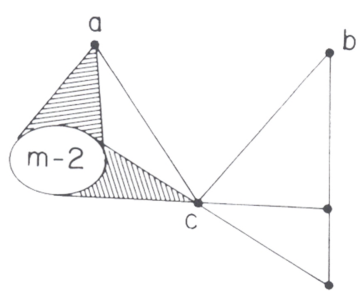

4

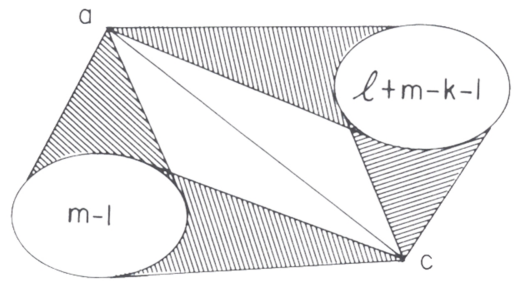

5

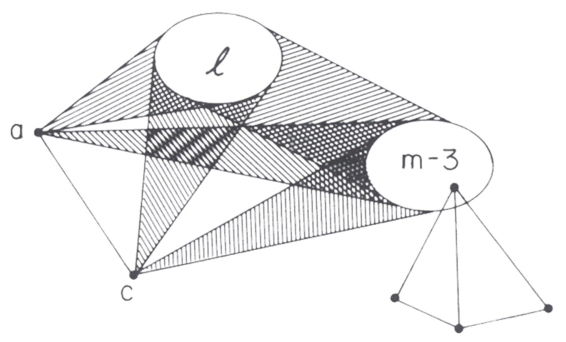

FIGURE 21 

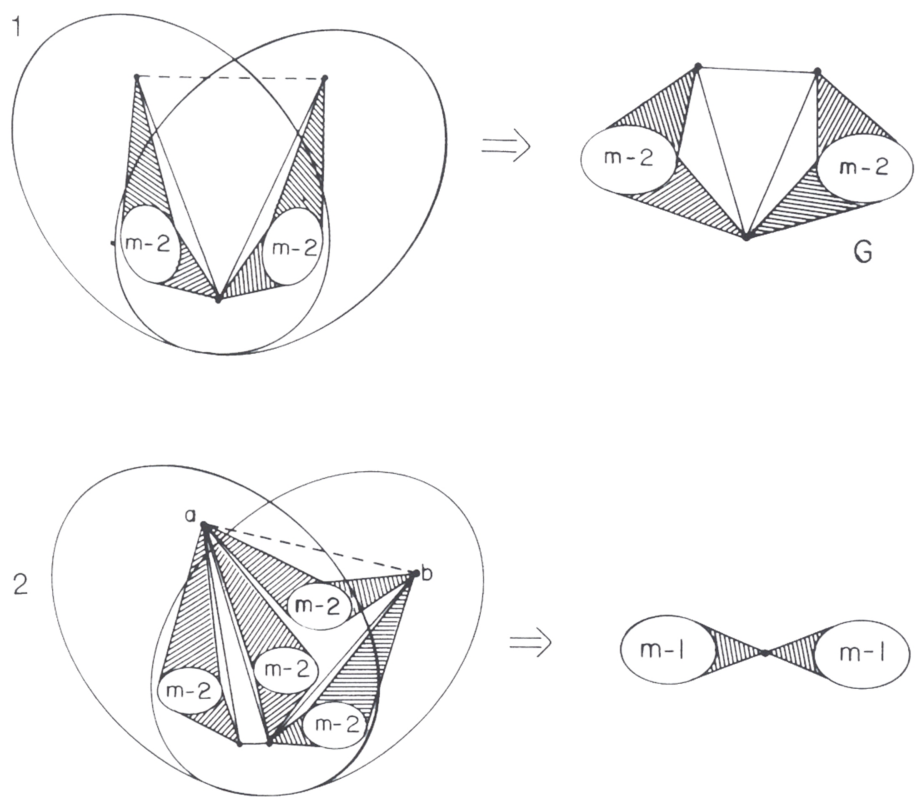

FiguRE 22
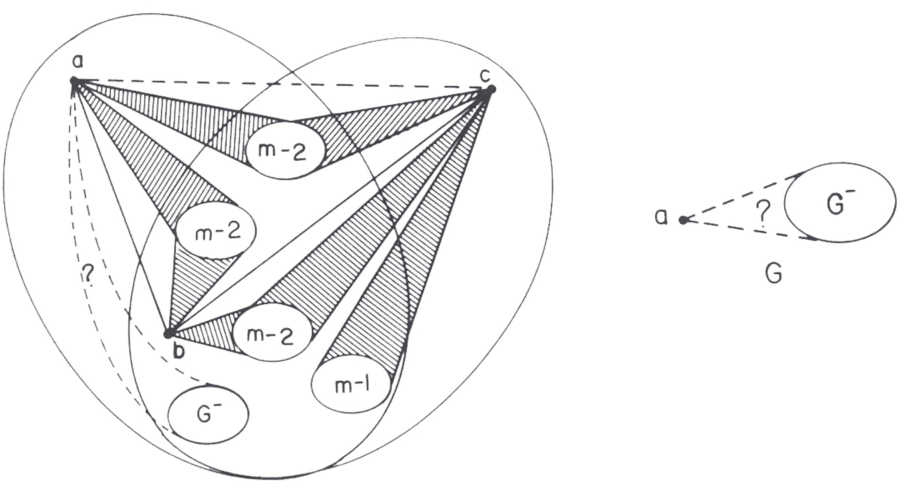

G

FigurE 23

(3) Again suppose $\vdash_{m}[(G, a),(K(m), a)]$ where $G$ is any graph, and $a$ is any vertex. We show that $\vdash_{m}[([(G, a),(K(m), a)], a),(K(m), a)]$, that is we can attach another copy of $K(m-1)$ to $a$.

From the amalgamation of Figure 24(1) we see that we have the graph we want with $a, b, K(m-2)$ if the edge $a, b$ is in the amalgamation and otherwise the graph $G_{\text {I }}$ of Figure 24(2). $G^{-}$results by deleting $a$ from $G$. In setting up this amalgamation we notice that the graph which results by deletion of $b$ is obtained from $[(G, a),(K(m), a)]$ by attaching successive copies of $K(m-2)$. With $a$ deleted we have a subgraph of the graph resulting from successive attachments of $K(m-2)$ to $G+K(m)+K(m-1)$. We can also obtain the graph $G_{\text {II }}$ of Figure 24(3) by first 
applying (2) twice to obtain the graph with $K(m-2)$ where $K(m-3)$ is written, and attaching $K(m-2)$ to $a^{\prime}$. Here $G^{\prime}$ is a copy of $G$ which is disjoint from $G . a^{\prime}$ is the image of $a$ under the isomorphism. $G^{\prime-}$ is the graph obtained from $G^{\prime}$ by deleting $a^{\prime}$. Now from the amalgamation of $G_{1}$ and $G_{1 I}$ shown in Figure 24(4) we obtain a graph in which some vertices of $G^{-}$and $G^{\prime-}$ may be identified, and there may or may not be edges from $G^{-}$to $\left(G^{\prime}\right)^{-}$. In any case $a$ and $a^{\prime}$ are distinct and no edge exists between $a$ and $a^{\prime}$. The exact outcome of the amalgamation does not affect the argument and we shall depict the outcome as in Figure 24(5). The cross-hatching on $G^{\prime},\left(G^{\prime}\right)^{-}$indicates that some edges or identifications between them may be made. Now by the amalgamation shown in Figure 24(6) of this graph with itself we obtain the result. A rigorous justification for this amalgamation is provided by the claim to Lemma D. If $H$ is $[([(G, a),(K(m), a)], a),(K(m), a)]$ then from Figure 24(1) we have $\vdash_{m} H \vee G_{I}$. The amalgamation of Figure 24(4) gives $\vdash_{m} H \vee G_{1} \vee \ldots \vee G_{k}$ where $G_{1}, \ldots, G_{k}$ enumerate the possible outcomes of this amalgamation. The amalgamation of Figure 24(6) shows that $\left\{G_{i}\right\} \vdash H$. This completes the proof of (3).

(4) Now by induction on $l$ we prove that $\vdash_{m} K(l) \otimes K(m-1)$ for $1<l<m$. From (1) and (2) it is obvious that $r_{m} K(2) \otimes K(m-1)$. Hence we assume that $3<l<m$ and that $r_{m} K(l-1) \otimes K(m-1)$. In proving that $\vdash K(l) \otimes K(m-1)$ we attach copies of $K(m-1)$ to the vertices of $K(l)$ one by one. The argument is much like that of part (3). Let us assume that we have attached $K(m-1)$ to $k$ vertices of $K(l)$ where $1 \leqslant k<l$ by part (1). The final amalgamation, which corresponds to Figure 24(6) is shown in Figure 25. The cross-hatching indicates that there may be some edges not shown between vertices in the top copies of $K(m-1)$ and those at the bottom. It is clear that this amalgamation will give us $k+1$ copies of $K(m-1)$ attached to the vertices of $K(l)$.

By the induction hypothesis we have the graph of Figure 26(1). By applications of (2) and (3) we may then derive the graph of Figure 26(2), in fact where $K(l)$ is replaced by $K(m)$.

Further, from the assumption that we can attach $K(m-1)$ to $k$ vertices of $K(l)$, and using (2) we have the graph of Figure 27 in $\mathcal{G}(m)$.

By amalgamating the graphs of Figure 26(2) and Figure 27 over their common part we then derive a disjunction of graphs of the general form shown in Figure 28. The cross-hatching indicates that some edges from the top copies of $K(m-1)$ to those at the bottom may be present. As in (3) this does not matter, and the symmetry of Figure 28 allows us to set up the amalgamation of Figure 25. This completes the proof of (4) and thus of (vi).

(vii) $\vdash_{m} L(3) \otimes K(m-1)$. Recall that we may attach $K(m-2)$ to any vertex in a derivation by (ii). Also from (vi) we have $\vdash_{m} K(m) \otimes K(m-1)$ and therefore $\vdash_{m} K(2) \otimes K(m-1)$. We consider two cases.

Case 1. $m=3$. Assume for contradiction $\forall_{3} L(3) \otimes K(2)$. By attaching $K(1)$ and forming disjoint unions we have the amalgamation of Figure 29(1). We are then in a position to do the amalgamation of Figure 29(2). When $c$ is deleted we have the graph of Figure 29(1). With $a, b$ deleted we have a subgraph of $5 K(3)$. If the edge 

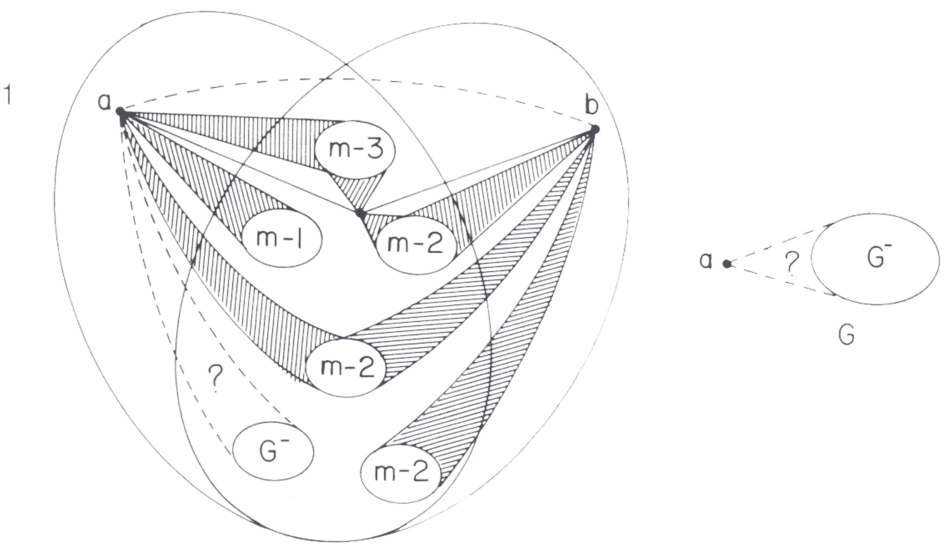

2

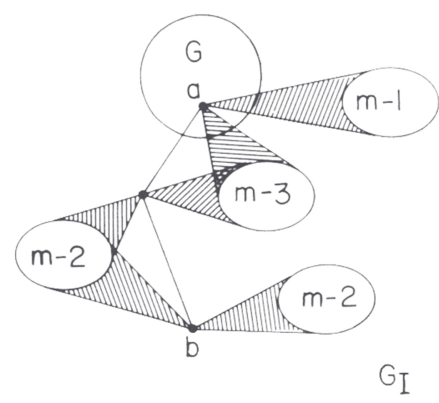

3

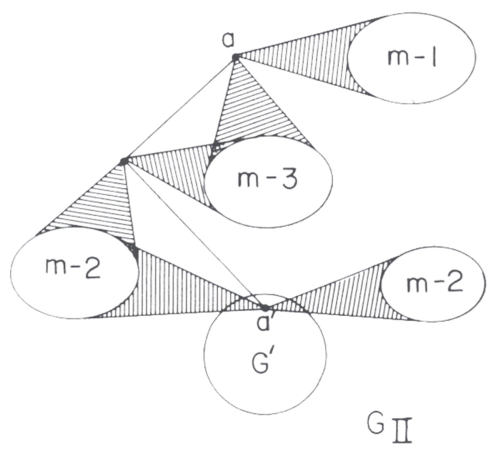

$a_{c}^{\prime}=-\frac{1}{G^{\prime}}$ 


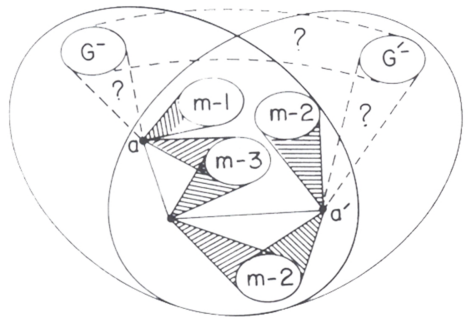

5

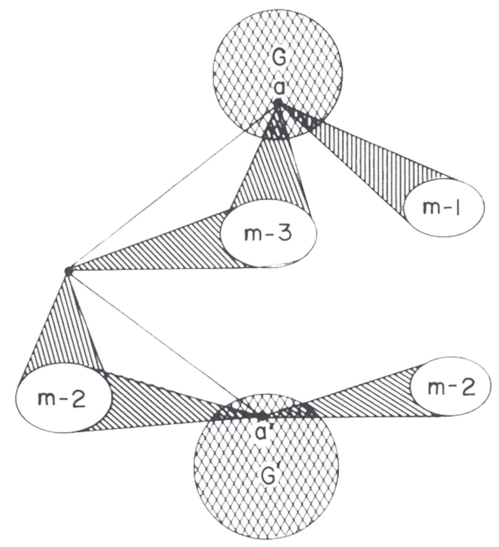

6

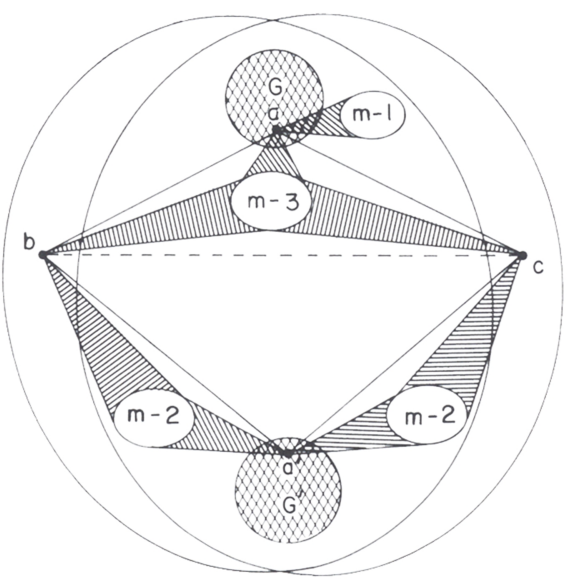

FIGURE 24 


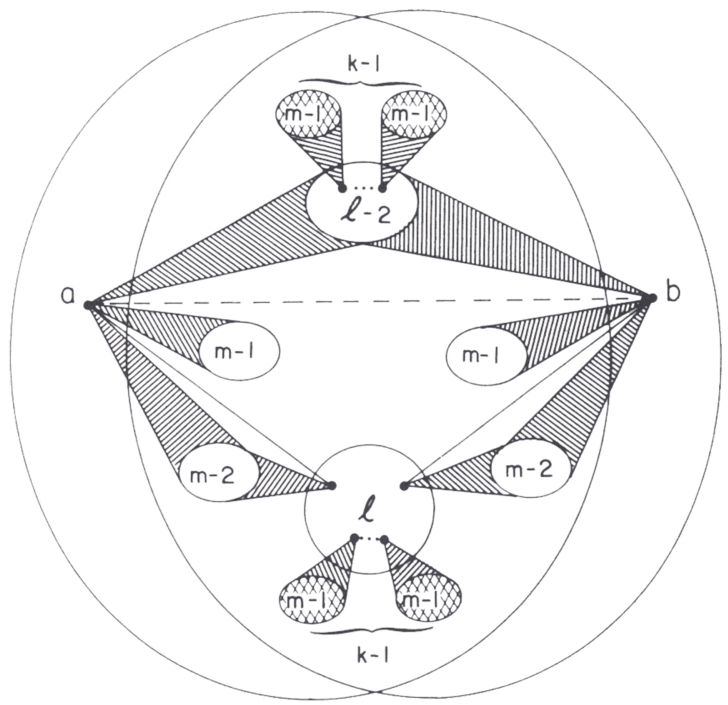

Figure 25
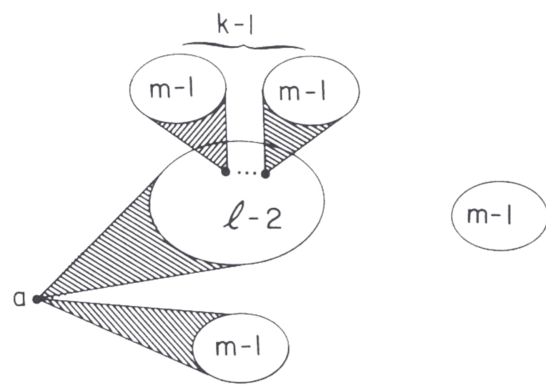

2

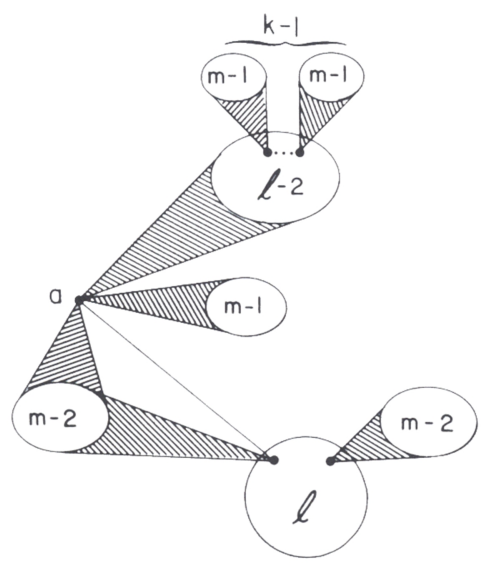

$m-1$

FigURE 26 


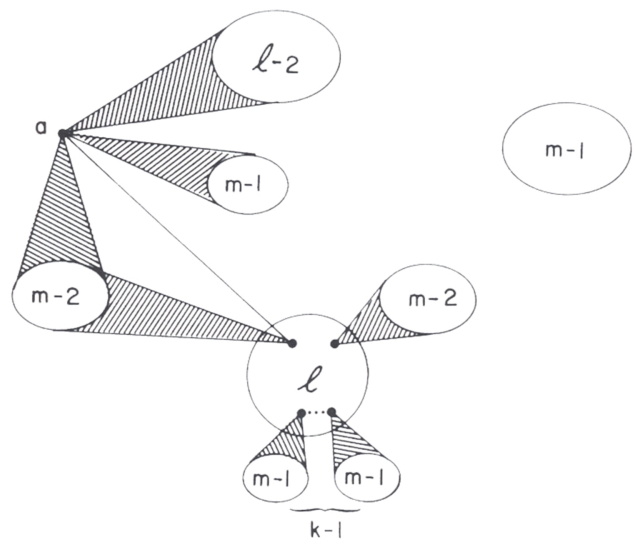

FIGURE 27

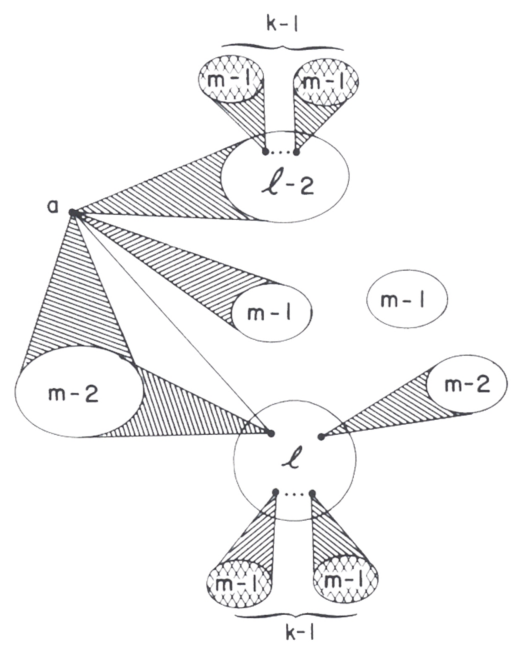

FigURE 28

$a, c$ is not present we are done with the top $K(2) \otimes K(2)$ since $c$ is then the third vertex of $K(3)$. Similarly the edge $b, c$ must be present. But then $a, d$ gives a copy of $K(2)$ suspended from $c$, and the edge $b, c$ connects this to the copy of $K(2) \otimes K(2)$. This shows that $r_{3} L(3) \otimes K(2)$.

Case 2. Let $m>3$. Assume for contradiction that $\forall_{m} L(3) \otimes K(m-1)$. Consider now the amalgamation of Figure 30. Deleting $c$ in Figure 30 we have a graph obtained from $K(2) \otimes K(m-1)$ by attaching a copy of $K(1)$ and two copies of $K(m-2)$ and forming the disjoint union with $2 K(m-1)$. With $a, b$ deleted we have a subgraph of the graph just obtained. If the edge $a, c$ is in the amalgamation we have $L(3) \otimes K(m-1)$ immediately. Now if the edge from $b$ to $c$ is in we again have $L(3) \otimes K(m-1)$ since $c, K(m-2)$ then form $K(m-1)$ suspended from $b$. Thus no edges are added. 


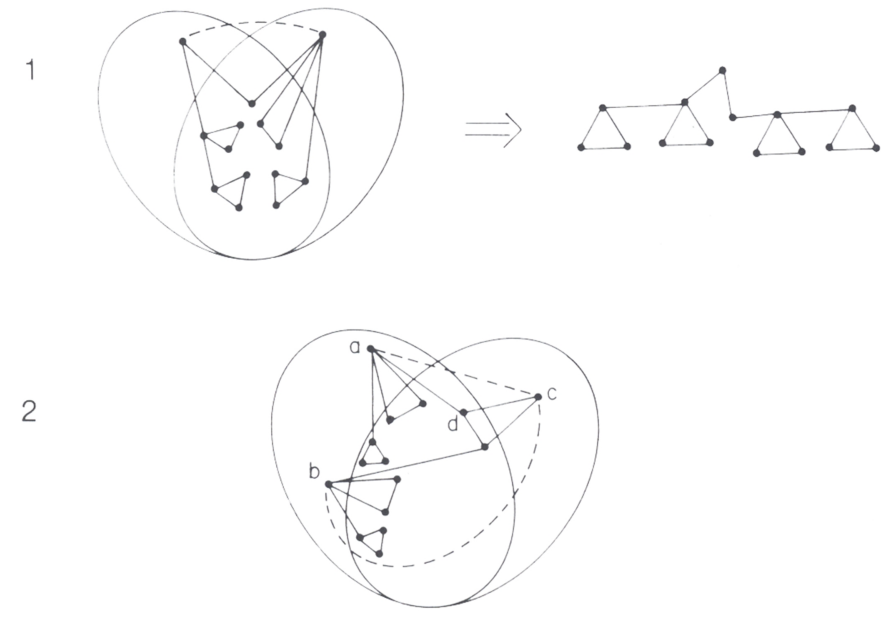

FiguRE 29

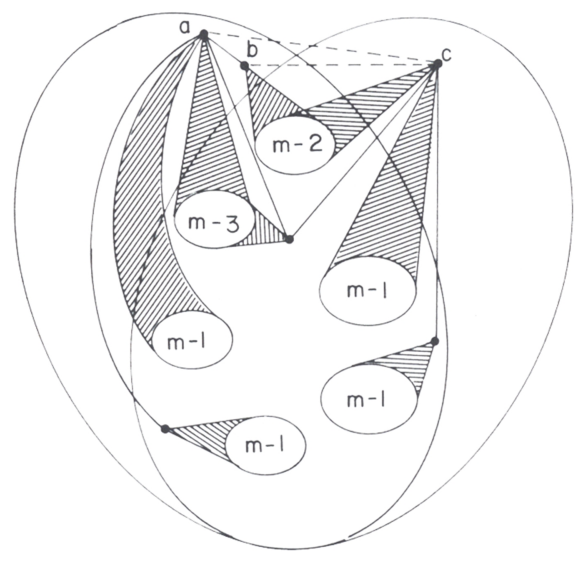

Figure 30

Now we obtain the desired contradiction by the amalgamation of Figure 31. With $e$ deleted we just have the graph of Figure 30. When $a, c$ are deleted we easily obtain the graph by attaching $K(m-2)$ to a vertex of $K(m)$ in $K(m)+$ $4 K(m-1)$. Unless the edge $a, e$ is in the amalgamation we are done for $e$, $K(m-2)$ give $K(m-1)$ suspended from $b$. There can be no edge from $c$ to $e$; observe that otherwise $c, e, K(m-2)$ give $K(m-1)$ suspended from $e$ and this is joined to $K(2) \otimes K(m-1)$ by the edge $a$, e. Finally $a, e, K(m-3)$ give $K(m-1)$ suspended from $d$ and connected by $c, d$ to the lower $K(2) \otimes K(m-1)$. Therefore $\vdash_{m} L(3) \otimes K(m-1)$ as required. 


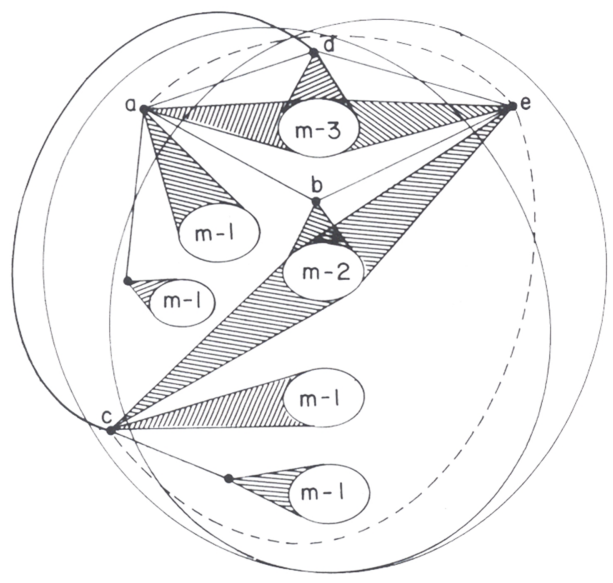

FigURE 31

(viii) Since $r_{3} K(3) \otimes K(2)$ and $r_{3} L(3) \otimes K(2)$ we have as in (ii) that if $r_{3} G_{1}$ $\vee \ldots \vee G_{n}$ then $\vdash_{3} G_{1} \otimes K(2) \vee \ldots \vee G_{n} \otimes K(2)$. Thus when $m=3$ we may add copies of $K(2)$ to vertices in any derivation.

(ix) $\vdash_{3} K(3) \otimes L(3)$. By (viii) we may freely add $K(1)$ and $K(2)$ to vertices. We have found no simple way to complete the proof of this part. One way that works is to repeat the technique of (vi). From (iv) we have $\vdash_{3} K(1) \otimes L(3)$. Now we obtain

$(1) \vdash_{3}[(\{a\} \otimes L(3), a),(\{a\} \otimes L(3), a)]$.

The amalgamations for this graph are shown in Figure 32. When there is no edge from $a$ to $b$ we use $a, d, b$ and when there is an edge from $a$ to $b$ we use $c, a, b$ to give the result.

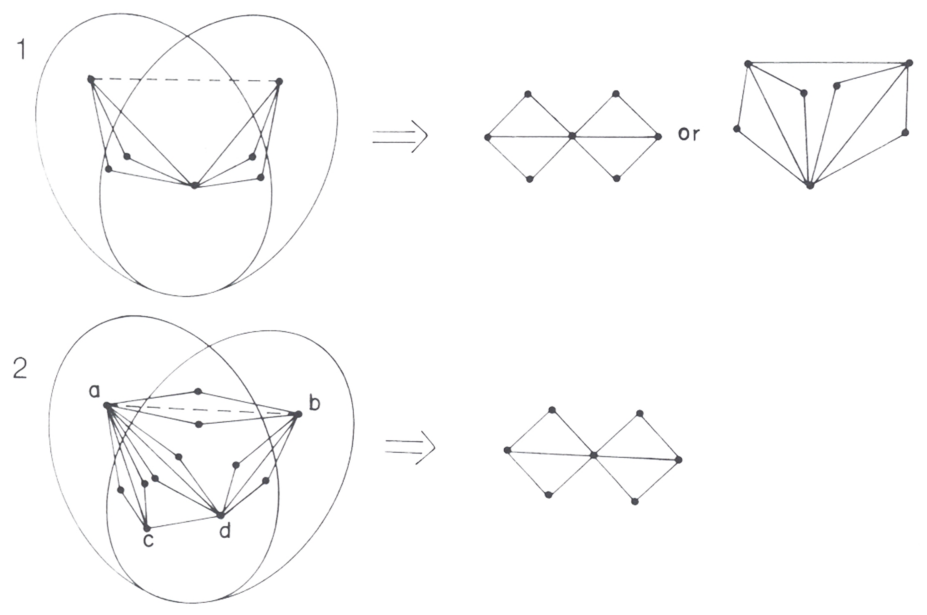


(2) If $\vdash_{3}[(G, a),(\{a\} \otimes L(3), a)]$ then $\vdash_{3}[([(G, a),(\{a\} \otimes L(3), a)], b),(\{b\} \otimes$ $L(3), b)]$,

where $b$ is the unique vertex of $\{a\} \otimes L(3)$ which is different from $a$ and such that there is an automorphism interchanging $a, b$. Let $G^{-}$result by deleting $a$ from $G$. The amalgamation which gives the result is shown in Figure 33. When $b$ is deleted the remaining graph may be obtained by attaching copies of $K(1)$ to the given graph. With $a$ deleted we have a graph which results from the disjoint union of $G^{-}$ and the graph of (1) by attaching copies of $K(1)$. This amalgamation clearly gives the result.
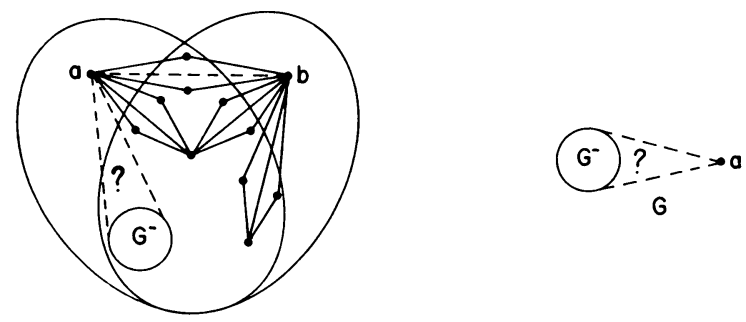

FIGURE 33

(3) If $\vdash_{3}[(G, a),(\{a\} \otimes L(3), a)]$ then $\vdash_{3}[([(G, a),(\{a\} \otimes L(3), a)], a),(\{a\} \otimes$ $L(3), a)]$.

Assume that $r_{3}[(G, a),(\{a\} \otimes L(3), a)]$. The amalgamation of Figure 34(1) uses the fact that we may attach a copy of $K(2)$ to $a . G^{-}$is the result of deleting $a$ from $G$. From this amalgamation we obtain the desired graph or the graph $G_{1}$ of Figure 34(2). We may obtain the graph of Figure 34(3) by two applications of (2). Here $G^{\prime}$ is an isomorphic copy of $G$ in which $a^{\prime}$ plays the role of $a . G^{\prime-}$ is the copy of $G^{-}$, i.e. the result of deleting $a^{\prime}$. We may now perform the amalgamation shown in Figure 34(4). In this amalgamation some identification of vertices of $G^{-}$and $G^{\prime-}$ may result, and some edges may be introduced. However $a, a^{\prime}$ are distinct and there is no edge from $a$ to $a^{\prime}$. The result of this amalgamation is shown in Figure 34(5). The cross-hatching indicates the possibility of identification and extra edges. We now exploit the symmetry of Figure 34(5) to set up the amalgamation shown in Figure 34(6). From this amalgamation we obtain the result. This completes the proof of (3).
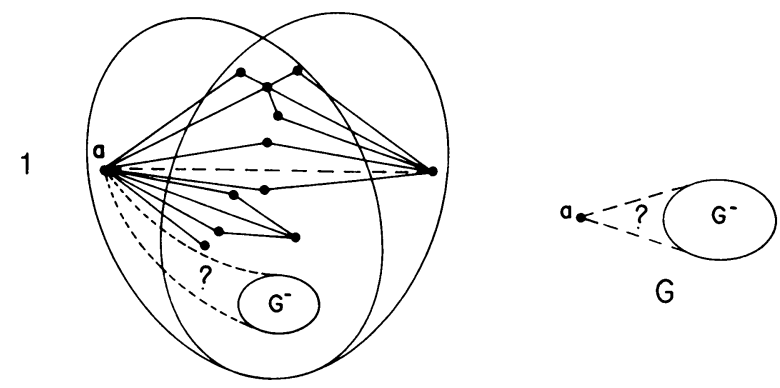
2

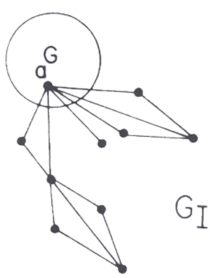

3
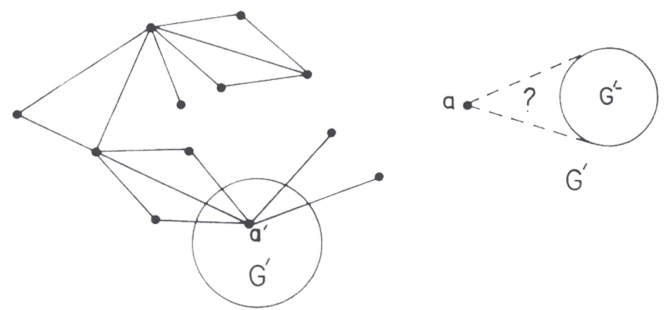

4

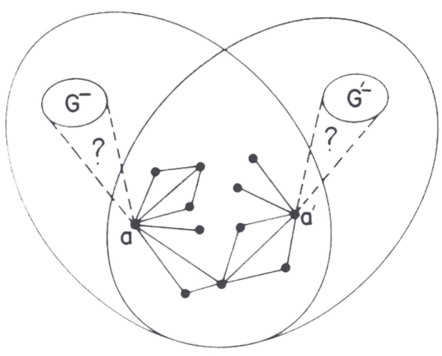

5

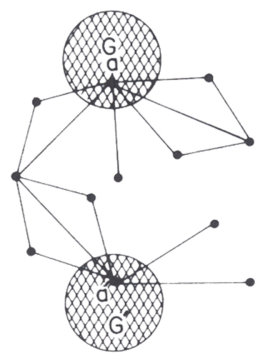

6

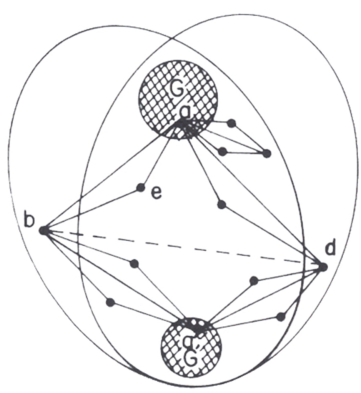

Figure 34 
(4) We now complete the proof of (ix) by showing $\vdash_{3} K(k) \otimes L(3)$ for $k=1,2,3$. We already have $r_{3} K(1) \otimes L(3)$. In fact from (1) and (2) we have $K(2) \otimes L(3)$. Applying (2) to $[(\{a\} \otimes L(3), a),(\{a\} \otimes L(3), a)]$ we have the graph of Figure 35 and $K(2) \otimes L(3)$ is a subgraph.

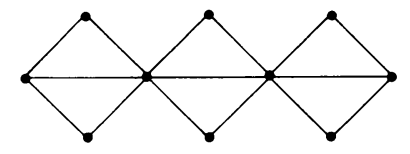

FigURE 35

Now we are in a position to perform the amalgamation of Figure 36(1). From $r_{3} K(2) \otimes L(3),(2),(3)$ and attaching copies of $K(1)$ we see that the graph of Figure $36(2)$ belongs to $\mathcal{G}(3)$. The symmetry of this situation permits the amalgamation of Figure 36(1). It is immediate from this that $r_{3} K(3) \otimes L(3)$ and (ix) follows.

1
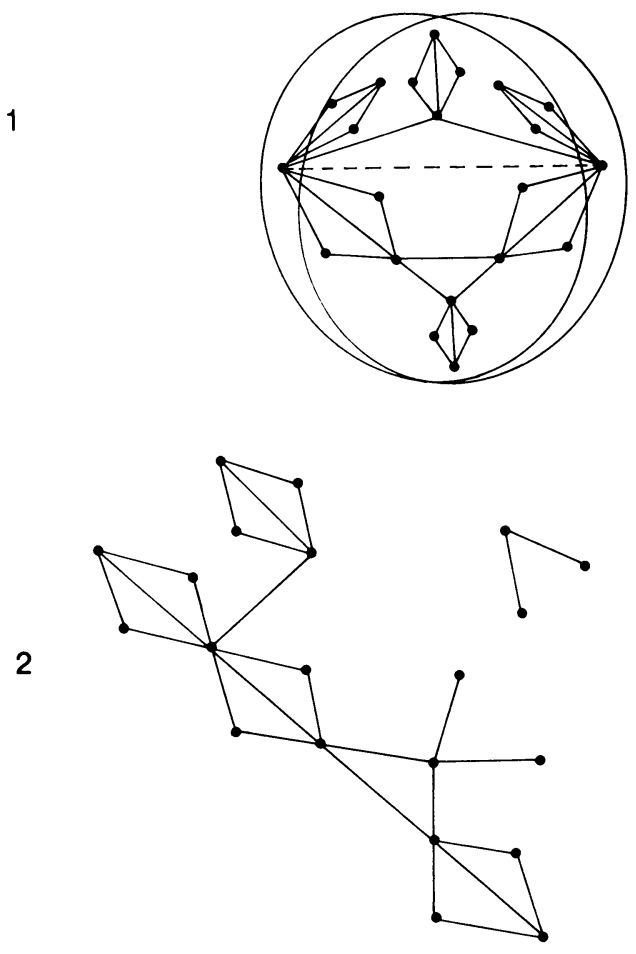

FigURE 36

(x) We now complete the proof of Lemma 3 by showing $\vdash_{3} L(3) \otimes L(3)$. Because we can attach copies of $K(2)$ it is clear from (ix) that we have the graph of Figure 37(1). This allows us to perform the amalgamation shown in Figure 37(2). This amalgamation gives us $L(3) \otimes L(3)$ if the edge $a, b$ is present and otherwise the graph $G$. The amalgamation of Figure 37(3) gives the graph $H$. With $G, H$ it is possible to set up the amalgamation of Figure 37(4). 
In this note that $a, c, e, f, g$ is the graph $H$. If there is not an edge from $d$ to $g$ we have $c, e, g$ forming the desired copy of $L(3)$ attached at $f$. Similarly if the edge from $b$ to $g$ is not in the amalgamation we have $e, f, g$ forming a copy of $L(3)$ attached to $c$ thus giving $L(3) \otimes L(3)$. But now with both edges $d, g$ and $b, g$ we have $a, b, c$ forming a copy of $L(3)$ attached to $g$, and this forms with the upper copy of $K(2) \otimes L(3)$ the desired graph. Thus $\vdash_{3} L(3) \otimes L(3)$.
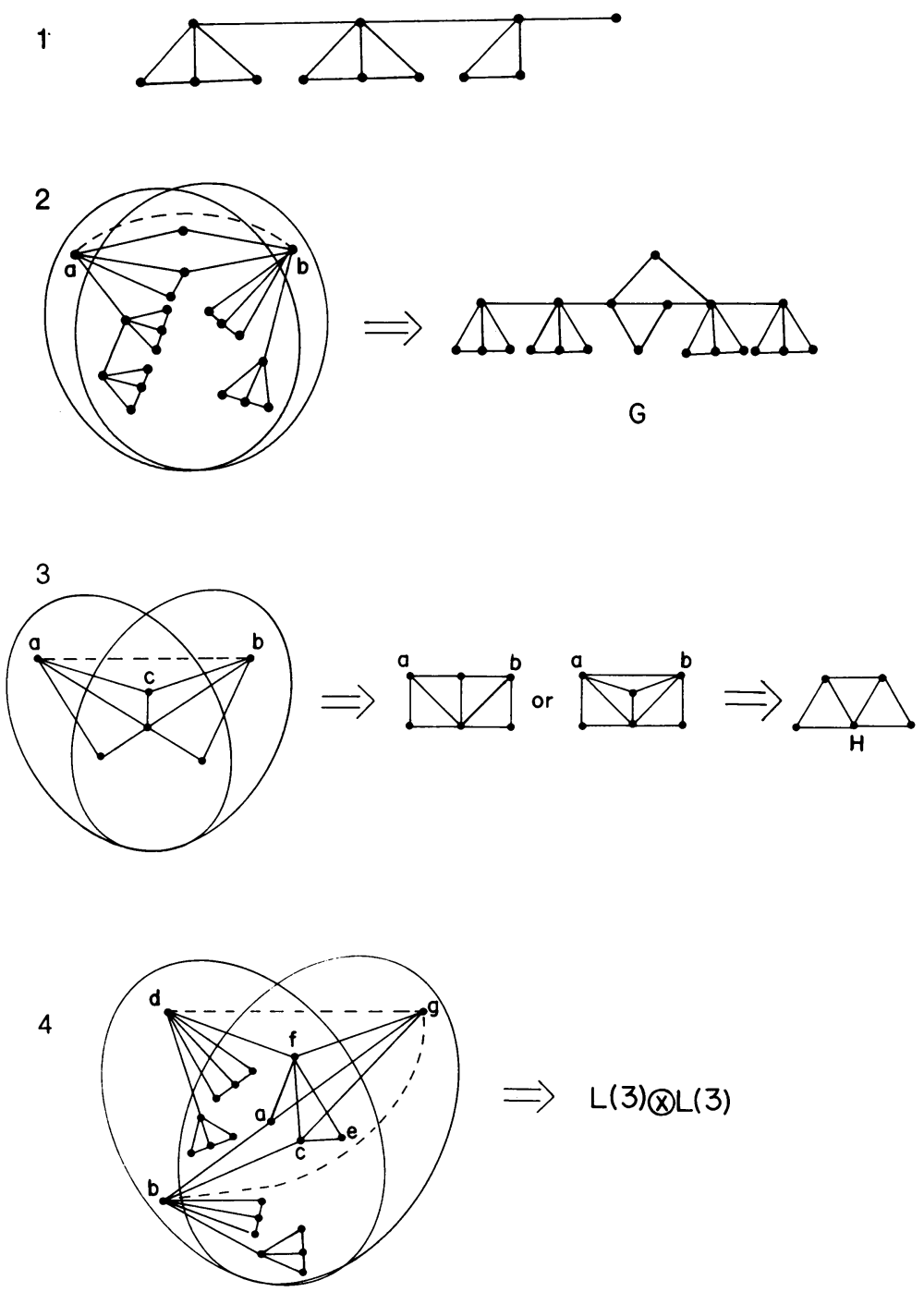

FIGURE 37

This completes the proof of Lemma 3. From Lemma 6, Theorem 2 is immediate because $\mathcal{G} *(m) \subset \mathcal{G}(m)$. 


\section{REFERENCES}

[F] R. Fraissé, Sur l'extension aux relations de quelques propriétés des ordres, Ann. Sci. École Norm. Sup. 71 (1954), 361-388.

[G] A. Gardiner, Homogeneous graphs, J. Combinatorial Theory Ser. B 20 (1976), 94-102.

[H1] C. Ward Henson, A family of countable homogeneous graphs, Pacific J. Math. 38 (1971), 69-83.

[H2] Countable homogeneous relational structures and $\aleph_{0}$-categorical theories, J. Symbolic Logic 37 (1972), 494-500.

[P] M. G. Peretyiatkin, On complete theories with a finite number of denumerable models, Algebra i Logika 12 (1973), 550-576.

[S] J. Schmerl, Countable homogeneous partial ordered sets, Algebra Universalis 9 (1979), 317-321.

[W1] Robert E. Woodrow, There are four countable ultrahomogeneous graphs without triangles, J. Combinatorial Theory Ser. B 27 (1979), 168-179.

[W2] Theories with a finite number of countable models and a small language, Ph.D. Dissertation, Simon Fraser University, 1976.

Department of Mathematics, Simon Fraser University, Burnaby, British Columbia, V5A 1 S6 Canada (Current address of A. H. Lachlan)

Current address (R. E. Woodrow): Department of Pure and Applied Mathematics and Statistics, University of Calgary, Calgary, Alberta T2N 1N4, Canada 